\title{
THE ALGEBRA OF POLYTOPES IN AFFINE SPACES
}

\section{BØRGE JESSEN and ANDERS THORUP}

\section{Introduction.}

In this paper we shall study the equivalence of polytopes with respect to the group of translations in finite dimensional affine spaces. The problem was studied and solved for dimension two by H. Hadwiger and P. Glur [11]. For dimension greater than two, necessary conditions for equivalence were discussed by $\mathrm{H}$. Hadwiger [3], [7], who proved their sufficiency for dimension three [8]. We shall prove that Hadwiger's conditions are sufficient in spaces of arbitrary dimension. Part of our treatment of the subject is merely a more algebraic exposition of the basic results of Hadwiger. We also obtain results about the structure of the group of equivalence classes; we show that it can be organized as a vector space, and that the dilation operator has a simple spectral decomposition.

Hadwiger considered polytopes in euclidean spaces, but might as well have considered affine spaces over an arbitrary ordered field. We have chosen to treat the subject in this generality. In order to make the exposition selfcontained, we have included brief proofs of the known results which we need.

The present investigation dates from 1972-73. Its principal results have been mentioned and used by $\mathrm{H}$. Hadwiger [9], [10] and P. Mürner [14], [15], [16], [17]. Another treatment of the subject has been given by C. H. Sah [18].

\section{Preliminaries.}

Let $L$ denote an ordered field, and $A$ an affine space over $L$ of dimension $k$ $\geqq 1$ with vector space $V$. In $A$ and $V$ we use the topologies induced by the order topology of $L$ When speaking of subspaces of $A$ and $V$ we normally mean subspaces of positive dimension.

By a polytope in $A$ we mean a subset of $A$ which is the union of a finite number of $k$-dimensional simplices whose interiors are disjoint.

A polytope $P$ in $A$ is said to be composed of the polytopes $P_{1}, \ldots, P_{n}$, or to be decomposed into $P_{1}, \ldots, P_{n}$, if $P=P_{1} \cup \ldots \cup P_{n}$, and the interiors of $P_{1}, \ldots, P_{n}$ are disjoint. If $P$ is composed of $P_{1}, \ldots, P_{n}$, we also say that $P$ is obtained from $P_{1}, \ldots, P_{n}$ by elementary addition, and if $P$ is composed of $P_{1}$

Received December 15, 1977. 
and $P_{2}$, we say that $P_{2}$ is obtained from $P$ and $P_{1}$ by elementary subtraction.

By the polytope group $\mathfrak{P}$ in $A$ we mean the free abelian group generated by the polytopes. By $\mathfrak{E}$ we denote the subgroup of $\mathfrak{P}$ generated by all elements $P$ $-P_{1}-P_{2}$, where $P$ is composed of $P_{1}$ and $P_{2}$, and all elements $P-Q$, where $Q$ is obtained from $P$ by a translation. Two elements $X$ and $Y$ of $\mathfrak{P}$ are called translation equivalent and we write $X \sim Y$, if $X-Y \in \mathfrak{E}$.

The group $\Pi=\mathfrak{P} / \mathfrak{E}$ of equivalence classes will be called the polytope group in $A$ modulo translations. The equivalence class containing an element $X$ of $\mathfrak{P}$ will be denoted by $[X]$. An element $x$ of $\Pi$ is said to be represented by the element $X$ of $\mathfrak{P}$, if $x=[X]$.

We shall be concerned with two problems: (1) to find necessary and sufficient conditions for two elements $X$ and $Y$ of $\mathfrak{P}$ to be translation equivalent, or, what amounts to the same, for an element $X$ of $\mathfrak{P}$ to be translation equivalent to 0 ; and (2) to discuss the structure of the group $\Pi$.

Instead of $\mathfrak{P}$ and $\mathfrak{E}$ we shall also write $\mathfrak{P}(A)$ and $\mathfrak{E}(A)$, and instead of $\Pi$ we shall also write $\Pi(A)$ or $\Pi(V)$. The latter notation is justified by the fact that there is a canonical identification between the group $\Pi$ and the polytope group modulo translations in $V$ (considered as an affine space). Indeed, by choosing a point $p_{0}$ in $A$, and considering the map $p \mapsto v$ of $A$ onto $V$ determined by $\overrightarrow{p_{0} p}$ $=v$, we obtain a bijection of $\mathfrak{P}(A)$ onto $\mathfrak{P}(V)$, which determines a bijection of $\Pi(A)$ onto $\Pi(V)$ independent of $p_{0}$.

Let $P_{1}, \ldots, P_{i}$ be polytopes in subspaces $A_{1}, \ldots, A_{i}$ of $A$, with corresponding vector spaces $V_{1}, \ldots, V_{i}$, such that $V=V_{1} \oplus \ldots \oplus V_{i}$. The product $P=P_{1} \times \ldots$ $\times P_{i}$, defined as the set of points in $A$ whose projection on each $A_{j}$ in the direction determined by the sum of the spaces $V_{h}, h \neq j$, belongs to $P_{j}$, is a polytope in $A$. Such a polytope $P$ is called an $i$-cylinder. If $P_{1}, \ldots, P_{i}$ are simplices, it is called an $i$-simplotope. A cylinder $I \times Q$, where $I$ is a simplex in a one-dimensional subspace, is called a prism with base $Q$. A $k$-simplotope is a parallelepipedon. Note that an $i$-cylinder is also a $j$-cylinder for any $j<i$. The map $\left(P_{1}, \ldots, P_{i}\right) \mapsto P_{1} \times \ldots \times P_{i}$ extends uniquely to a map, denoted $\left(X_{1}, \ldots, X_{i}\right) \mapsto X_{1} \times \ldots \times X_{i}$, from the cartesian product of $\mathfrak{P}\left(A_{1}\right), \ldots, \mathfrak{P}\left(A_{i}\right)$ into $\mathfrak{B}$, which is additive in each variable. The equivalence class $\left[X_{1} \times \ldots\right.$ $\left.\times X_{i}\right]$ depends only on the equivalence classes $\left[X_{1}\right], \ldots,\left[X_{i}\right]$. It will be called the product of $\left[X_{1}\right], \ldots,\left[X_{i}\right]$ and denoted by $\left[X_{1}\right] \times \ldots \times\left[X_{i}\right]$. The map $\left(x_{1}, \ldots, x_{i}\right) \mapsto x_{1} \times \ldots \times x_{i}$ from the cartesian product of $\Pi\left(A_{1}\right), \ldots, \Pi\left(A_{i}\right)$ into $\Pi$ is additive in each variable.

The subgroup of $\mathfrak{P}$ generated by $\mathfrak{E}$ and the $i$-cylinders will be denoted by $\mathbf{Z}_{i}$, and the quotient group $Z_{i} / \mathfrak{E}$ by $Z_{i}$. In this way we obtain the filtrations

$$
\begin{aligned}
& \mathfrak{P}=\mathbf{Z}_{1} \supseteqq \mathcal{3}_{2} \supseteqq \ldots \supseteqq \mathcal{Z}_{k} \supseteqq \mathfrak{E} \\
& \Pi=Z_{1} \supseteqq Z_{2} \supseteqq \ldots \supseteqq Z_{k} \supseteqq\{0\}=Z_{k+1} .
\end{aligned}
$$


The notation $Z_{k+1}$ for $\{0\}$ is introduced for convenience. Instead of $\mathcal{Z}_{i}$ we shall also write $Z_{i}(A)$, and instead of $Z_{i}$ we shall also write $Z_{i}(A)$ or $Z_{i}(V)$.

For arbitrary linearly independent vectors $a_{1}, \ldots, a_{k}$ in $V$ we denote by $\left[a_{1}, \ldots, a_{k}\right]$ the equivalence class which, for an arbitrary point $p_{0}$, contains the simplex with vertices $p_{0}, p_{1}, \ldots, p_{k}$, where $\overrightarrow{p_{j-1} p_{j}}=a_{j}$ for $j \in\{1, \ldots, k\}$. Combining this notation with the notation for a product, we obtain for the equivalence class containing an $i$-simplotope the notation $\left[a_{1}, \ldots, a_{j_{1}}\right] \times \ldots$ $\times\left[a_{j_{t-1}+1}, \ldots, a_{k}\right]$, where $a_{1}, \ldots, a_{k}$ are linearly independent vectors, and $1 \leqq j_{1}$ $<\ldots<j_{i-1}<k$.

Let $\mathfrak{5}$ be an arbitrary abelian group. Every mapping from the class of polytopes into $\mathfrak{b}$ can be uniquely extended to a homomorphism from $\mathfrak{P}$ into $\mathfrak{b}$. Homomorphisms $f: \mathfrak{P} \rightarrow \mathfrak{G}$, which vanish on $\mathfrak{E}$, or, what amounts to the same, homomorphisms $f: \Pi \rightarrow \mathfrak{G}$, correspond uniquely to mappings $P \mapsto f(P)$ from the class of polytopes into $(5$ which are additive and translation invariant, i.e., for which $f(P)=f\left(P_{1}\right)+f\left(P_{2}\right)$, when $P$ is composed of $P_{1}$ and $P_{2}$, and $f(P)=f(Q)$, when $Q$ is obtained from $P$ by a translation. Note that we use the same notation for a homomorphism from $\mathfrak{P}$ into $\mathfrak{G}$, which vanishes on $\mathfrak{E}$, and for the corresponding homomorphism from $\Pi$ into $\mathbf{b}$.

It is known that, in order to define an additive and translation invariant mapping from the class of polytopes into $\mathfrak{G}$ it is enough to define a translation invariant mapping from the class of simplices into $\mathfrak{5}$, which is additive with respect to decompositions of a simplex into two simplices by a $(k-1)$ dimensional subspace through $k-1$ of its vertices.

The existence of homomorphisms $f: \Pi \rightarrow L$, which do not vanish identically, will be proved in section 2 . It implies that $\Pi \neq\{0\}$, and also that the endomorphism ring End $\Pi$ of the abelian group $\Pi$ contains a copy of $Z$.

With every automorphism $\varphi$ of the vector space $V$ there is associated an automorphism $\bar{\varphi}$ of $\Pi$, with the property that $\bar{\varphi}([P])=[\alpha(P)]$ for the automorphisms $\alpha$ of $A$ for which $\overrightarrow{\alpha(p) \alpha(q)}=\varphi(\overrightarrow{p q})$ for all $p, q \in A$. For any two automorphisms $\varphi$ and $\psi$ of $V$, we have $\overline{\varphi \psi}=\bar{\varphi} \bar{\psi}$. The subgroups $Z_{i}$ are stable under the maps $\bar{\varphi}$.

Denoting by $\delta(\lambda)$ for $\lambda \in L \backslash\{0\}$ the homothesi in $V$ defined by $\delta(\lambda) a=\lambda a$, we define the dilation operator $d(\hat{\lambda})$ in $\Pi$ by $d(\hat{\lambda})=\overline{\delta(\lambda)}$, when $\lambda>0$, and $d(\hat{\lambda})=$ $(-1)^{k} \overline{\delta(\hat{\lambda})}$, when $\hat{\lambda}<0$. Furthermore, we define $d(0)$ as 0 . Note that $d(1)=1$. Note also that $d(\lambda)$ commutes with $\bar{\varphi}$ for every automorphism $\varphi$ of $V$. The introduction of the sign $(-1)^{k}$ in the definition of $d(\lambda)$ for $\lambda<0$ connects with the fact that we do not work with an oriented space and oriented polytopes. The function $d: L \rightarrow$ End $\Pi$ is multiplicative, i.e., $d(\lambda \mu)=d(\hat{\lambda}) d(\mu)$ for arbitrary $\hat{\lambda}, \mu \in L$.

The notation $d(\lambda)$ will be used also for the dilation operator in the polytope groups modulo translations belonging to subspaces of $A$. 
Let $P$ be a polytope, let $B$ be a $(k-1)$-dimensional subspace of $A$, and $\bar{B}$ one of the open halfspaces bounded by $B$. We say that $P$ has a face in $B$ corresponding to $\bar{B}$, if there are points $p$ on the boundary of $P$ for which the intersection of $\bar{B}$ and a neighbourhood of $p$ is contained in $P$. The closure of the set of these points $p$ is the face of $P$ in $B$ corresponding to $\bar{B}$; it is a polytope in $B$. It is called negative or positive with respect to a vector $e$ not in $B$, according as $\bar{B}$ is or is not the halfspace into which $e$ is pointing. Note that each face $F$ of $P$ determines a unique $(k-1)$-dimensional subspace $B$ of $A$ and a unique halfspace $\bar{B}$ bounded by $B$, such that $F$ is the face of $P$ in $B$ corresponding to $\bar{B}$.

Remark. Two polytopes $P$ and $Q$ are called "zerlegungsgleich" modulo translations, and we write $P \approx Q$, if there are decompositions $P=P_{1} \cup \ldots \cup P_{n}$ and $Q=Q_{1} \cup \ldots \cup Q_{n}$ such that $Q_{i}$ is a translate of $P_{i}$ for every $i \in\{1, \ldots, n\}$. Obviously, if $P \approx Q$, then $P \sim Q$. But conversely, from $P \sim Q$ it follows only that $P$ and $Q$ are "ergänzungsgleich" modulo translations, i.e., that there exist polytopes $R \approx S$ and $\bar{R} \approx \bar{S}$, such that $\bar{R}$ is composed of $P$ and $R$ and $\bar{S}$ is composed of $Q$ and $S$. However, "ergänzungsgleich" implies "zerlegungsgleich", if the ordered field $L$ is archimedean, cf. J.-P. Sydler [19], H. Hadwiger [4], V. B. Zylev [20], and V. G. Boltjanskiǐ [1].

\section{The equivalence theorem. First part.}

Let $S_{0}$ denote an arbitrarily chosen simplex in $A$. For an arbitrary simplex $S$ in $A$, there are $(k+1)$ ! automorphisms of $A$ which take $S_{0}$ into $S$. The absolute values of their determinants are equal. The function from the class of simplices into $L$, whose value for a simplex $S$ is the absolute value of these determinants, is translation invariant, and additive with respect to decompositions of a simplex into two simplices by a $(k-1)$-dimensional subspace through $k-1$ of its vertices. It therefore defines a group homomorphism $\mathrm{Vol}_{k}: \mathfrak{P} \rightarrow L$ which vanishes on $\mathfrak{E}$. We call this homomorphism, and also the corresponding homomorphism $\mathrm{Vol}_{k}: \Pi \rightarrow L$, the $k$-dimensional volume with respect to $S_{0}$. Its value for a polytope is positive. The volumes corresponding to different choices of $S_{0}$ are proportional. When we speak of the volume, it will be understood to be with respect to a chosen $S_{0}$. When we speak of the volume $\mathrm{Vol}_{i}$ for $i$ dimensional subspaces of $A$, it will be understood to be with respect to simplices that for parallel subspaces are translates of each other, whereas we make no assumption connecting the volumes for subspaces that are not parallel.

The vanishing of $\mathrm{Vol}_{k}: \mathfrak{P} \rightarrow L$ on $\mathbb{E}$ means that the condition $\mathrm{Vol}_{k} X=0$ is 
necessary for the equivalence $X \sim 0$. If $k=1$, this condition is also sufficient. For $k>1$, further conditions are obtained as follows.

Let $A^{k-1}$ be any $(k-1)$-dimensional subspace of $A$, let $V^{k-1}$ be the corresponding subspace of $V$, and let $e^{k-1}$ be a vector not in $V^{k-1}$. We consider the homomorphism

$$
h\left(A^{k-1}, e^{k-1}\right): \mathfrak{P} \rightarrow \mathfrak{P}\left(A^{k-1}\right)
$$

whose value for a polytope $P$ is defined in the following way: Consider those faces $F$ of $P$ which are parallel to $A^{k-1}$, and denote by $F^{\prime}$ the projection of such a face on $A^{k-1}$ in the direction determined by $e^{k-1}$. Then $h\left(A^{k-1}, e^{k-1}\right) P$ is the sum $\sum \varepsilon F^{\prime}$ extended over all these faces, where for each face $F$ the factor $\varepsilon$ is +1 or -1 according as the face is positive or negative with respect to $e^{k-1}$. One sees immediately that if $X \sim 0$, then $h\left(A^{k-1}, e^{k-1}\right) X \sim 0$. This necessary condition for the equivalence $X \sim 0$ means that $h\left(A^{k-1}, e^{k-1}\right)$ defines a group homomorphism from $\mathfrak{P}$ into $\Pi\left(V^{k-1}\right)$ vanishing on $\mathfrak{E}$, and hence a group homorphism from $\Pi$ into $\Pi\left(V^{k-1}\right)$. This group homomorphism is easily seen to be the same for all subspaces $A^{k-1}$ of the same direction $V^{k-1}$, and all vectors $e^{k-1}$ lying at the same side of $V^{k-1}$, whereas for vectors $e^{k-1}$ at different sides of $V^{k-1}$ the homomorphisms differ by a factor -1 . Thus we have essentially only one condition for each $V^{k-1}$. Note that, for each $X \in \mathfrak{P}$, the condition $h\left(A^{k-1}, e^{k-1}\right) X \sim 0$ is trivially satisfied for all but a finite number of subspaces $V^{k-1}$, namely for those subspaces $V^{k-1}$ for which there are no faces of direction $V^{k-1}$ in the polytopes generating $X$.

It was proved for $k=2$ by $\mathrm{H}$. Hadwiger and P. Glur [11] and for $k=3$ by H. Hadwiger [8] that these conditions together with the condition $\operatorname{Vol}_{k} X=0$ are also sufficient for the equivalence $X \sim 0$. We shall prove in section 6 that they are sufficient for all $k$.

This gives the first form of the equivalence theorem:

THEOREM 1. An element $X$ of the polytope group $\mathfrak{P}(A)$ is translation equivalent to 0 , if and only if $\operatorname{Vol}_{k} X=0$, and, for any $(k-1)$-dimensional subspace $A^{k-1}$ of $A$ and any vector $e^{k-1}$ not parallel to $A^{k-1}$, the element $h\left(A^{k-1}, e^{k-1}\right) X$ of the polytope group $\mathfrak{P}\left(A^{k-1}\right)$ is translation equivalent to 0 .

Applying the necessity of the conditions in Theorem 1 for dimension $k-1$, one sees that the condition $h\left(A^{k-1}, e^{k-1}\right) X \sim 0$ implies that $\operatorname{Vol}_{k-1} h\left(A^{k-1}, e^{k-1}\right) X=0$, and also that for any $(k-2)$-dimensional subspace $A^{k-2}$ of $A^{k-1}$ and any vector $e^{k-2}$ parallel to $A^{k-1}$ but not to $A^{k-2}$, we have

$$
h\left(A^{k-2}, e^{k-2}\right) h\left(A^{k-1}, e^{k-1}\right) X \sim 0 .
$$

Proceeding in this manner, one arrives at a system of necessary conditions for the equivalence $X \sim 0$, involving volumes in subspaces of all dimensions. These conditions can be formulated as follows. 
Consider descending sequences $V \supset V^{k-1} \supset \ldots \supset V^{i}$, where each $V^{j}$ is a subspace of $V$ of dimension $j$. For each such sequence, let $\overline{V^{j}}$ for every $j$ be one of the two open halfspaces of $V^{j+1}$ bounded by $V^{j}$ (here $V^{k}$ means $V$ ). The sequence $\Phi=\left(V, \overline{V^{k-1}}, \ldots, \overline{V^{i}}\right)$ will be called an $i$-flag in $V$. With each flag $\Phi$ we now associate a Hadwiger function $H_{\Phi}: \mathfrak{P} \rightarrow L$. There is only one $k$-flag, viz. $\Phi$ $=(V)$. For this we define the Hadwiger function as $H_{\Phi}=\operatorname{Vol}_{k}$. For an $i$-flag $\Phi$, where $i<k$, we define the Hadwiger function by

$$
H_{\Phi}(X)=\operatorname{Vol}_{i} h\left(A^{i}, e^{i}\right) \ldots h\left(A^{k-1}, e^{k-1}\right) X,
$$

where each $A^{j}$ is a $j$-dimensional subspace of $A$ of direction $V^{j}$, and each $e^{j}$ is a vector in $\overline{V^{j}}$. From the remarks made above it is clear that the volume on the right hand side for a given $X$ depends only on $\Phi$. The function $H_{\Phi}: \mathfrak{P} \rightarrow L$ is a group homomorphism vanishing on $\mathfrak{E}$. The necessary conditions for the equivalence $X \sim 0$ can now be written $H_{\Phi}(X)=0$. For two flags $\Phi$ and $\Psi$ corresponding to the same sequence $V \supset V^{k-1} \supset \ldots \supset V^{i}$ the functions $H_{\Phi}$ and $H_{\psi}$ are either identical or they differ by a factor -1 . Thus we have essentially only one condition for each sequence $V \supset V^{k-1} \supset \ldots \supset V^{i}$. Note that, for each $X \in \mathfrak{P}$, the condition $H_{\Phi}(X)=0$ is trivially satisfied for all but a finite number of flags. Note further that, for each flag $\Phi$, there are simplices $S$ for which $H_{\Phi}(S)$ $\neq 0$.

We can now state the second form of the equivalence theorem:

THEOREM 2. An element $X$ of the polytope group $\mathfrak{P}(A)$ is transtation equivalent to 0 if and only if $H_{\Phi}(X)=0$ for all flags $\Phi$.

For $k=1$ the two theorems are identical. Since the conditions in Theorem 2 follow from the conditions in Theorem 1, it is evident that if the conditions of Theorem 2 are sufficient, then the conditions of Theorem 1 are also sufficient. The converse is easily proved by induction after the dimension.

Note that the Hadwiger function $H_{\Phi}$ for an $i$-flag $\Phi$, considered as a function $H_{\Phi}: \Pi \rightarrow L$, is homogeneous of degree $i$ with respect to the dilation operator, that is to say that for every $x \in \Pi$ and every $\lambda \in L$ we have $H_{\Phi}(d(\lambda) x)=\lambda^{i} H_{\Phi}(x)$.

\section{Basic geometrical lemmas.}

Our treatment of the algebra of polytopes depends on two lemmas on simplices and simplotopes.

LemMA 1. For arbitrary linearly independent vectors $a_{1}, \ldots, a_{k}$ and arbitrary $\lambda, \mu \in L$ we have

$$
d(\lambda+\mu)\left[a_{1}, \ldots, a_{k}\right]=\sum_{j=0}^{k} d(\mu)\left[a_{1}, \ldots, a_{j}\right] \times d(\lambda)\left[a_{j+1}, \ldots, a_{k}\right] .
$$


The terms corresponding to $j=0$ and $j=k$ shall mean $d(\lambda)\left[a_{1}, \ldots, a_{k}\right]$ and $d(\mu)\left[a_{1}, \ldots, a_{k}\right]$, respectively.

Proof. If $\lambda=0$ or $\mu=0$, the formula is obvious. Assume now that $\lambda \neq 0, \mu \neq 0$. From the validity of the formula when $\lambda+\mu>0$ follows its validity when $\lambda+\mu$ $<0$ by replacing $\lambda, \mu$ by $-\lambda,-\mu$. From its validity when $\lambda<0, \mu>0$ follows its validity when $\lambda>0, \mu<0$ by writing $\left[-a_{k}, \ldots,-a_{1}\right]$ instead of $\left[a_{1}, \ldots, a_{k}\right]$. It is therefore sufficient to consider the cases $\lambda>0, \mu>0$ and $\lambda<0, \lambda+\mu \geqq 0$.

(i) $\lambda>0, \mu>0$ (see fig. 1). In a coordinate system with $a_{1}, \ldots, a_{k}$ as base vectors, $d(\lambda+\mu)\left[a_{1}, \ldots, a_{k}\right]$ is represented by the simplex

$$
S=\left\{\left(t_{1}, \ldots, t_{k}\right) \mid 0 \leqq t_{k} \leqq \ldots \leqq t_{1} \leqq \lambda+\mu\right\},
$$

and the $j$ th term of the sum is represented by the polytope

$$
P_{j}=\left\{\left(t_{1}, \ldots, t_{k}\right) \mid 0 \leqq t_{k} \leqq \ldots \leqq t_{j+1} \leqq \lambda \leqq t_{j} \leqq \ldots \leqq t_{1} \leqq \lambda+\mu\right\} .
$$

Since $S$ is composed of $P_{0}, \ldots, P_{k}$, the formula follows.
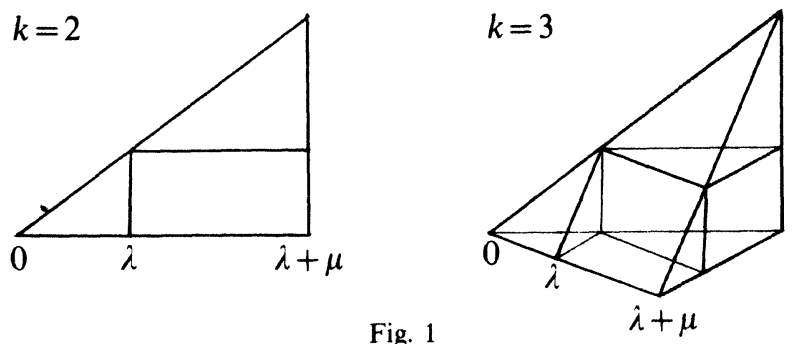

Fig. 1

(ii) $\lambda<0, \lambda+\mu>0$ (see fig. 2). In a coordinate system with $a_{1}, \ldots, a_{k}$ as base vectors, $d(\lambda+\mu)\left[a_{1}, \ldots, a_{k}\right]$ is represented by the simplex

$$
S=\left\{\left(t_{1}, \ldots, t_{k}\right) \mid 0 \leqq t_{k} \leqq \ldots \leqq t_{1} \leqq \lambda+\mu\right\} .
$$

The $j$ th term of the sum is now $(-1)^{k-j}\left[P_{j}\right]$, where $P_{j}$ is the polytope

$$
P_{j}=\left\{\left(t_{1}, \ldots, t_{k}\right) \mid \lambda \leqq t_{j} \leqq \ldots \leqq t_{1} \leqq \lambda+\mu, \lambda \leqq t_{j+1} \leqq \ldots \leqq t_{k} \leqq 0\right\} .
$$

Consider for $0<j<k$ the polytopes

$$
Q_{j}=\left\{\left(t_{1}, \ldots, t_{k}\right) \mid t_{j+1} \leqq t_{j} \leqq \ldots \leqq t_{1} \leqq \lambda+\mu, \lambda \leqq t_{j+1} \leqq \ldots \leqq t_{k} \leqq 0\right\},
$$

and put $Q_{0}=P_{0}, Q_{k}=S$. Since $P_{j}$ for $0<j \leqq k$ is composed of $Q_{j-1}$ and $Q_{j}$, we find

$$
\sum_{j=0}^{k}(-1)^{k-j}\left[P_{j}\right]=(-1)^{k}\left[Q_{0}\right]+\sum_{j=1}^{k}(-1)^{k-j}\left(\left[Q_{j-1}\right]+\left[Q_{j}\right]\right)=\left[Q_{k}\right]
$$



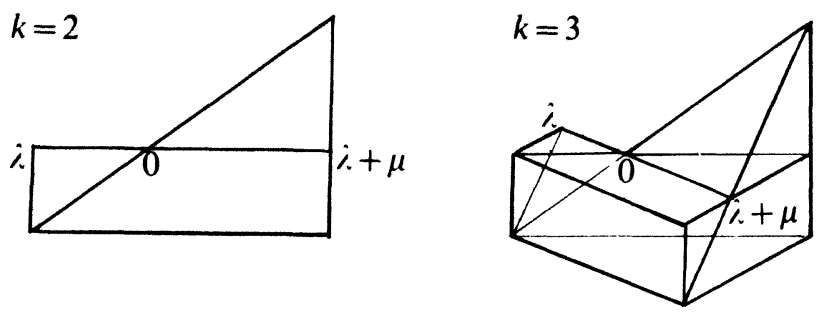

Fig. 2

(iii) $\lambda<0, \lambda+\mu=0$. The proof is as in the preceding case, with 0 instead of $S$.

Corollary. From Lemma 1 it follows that

$$
d(\lambda+\mu)\left[a_{1}, \ldots, a_{k}\right] \equiv d(\dot{\lambda})\left[a_{1}, \ldots, a_{k}\right]+d(\mu)\left[a_{1}, \ldots, a_{k}\right] \bmod Z_{2} .
$$

Hence, for every $n \in \mathbb{N}$,

$$
d(n)\left[a_{1}, \ldots, a_{k}\right]=\left[n a_{1}, \ldots, n a_{k}\right] \equiv n\left[a_{1}, \ldots, a_{k}\right] \bmod Z_{2} .
$$

Lemma 2. For arbitrary linearly independent vectors $a_{1}, \ldots, a_{k}$ and arbitrary $\lambda, \mu \in L$ we have for every $j \in\{1, \ldots, k-1\}$

$$
\begin{aligned}
d(\hat{\lambda})\left[a_{1}, \ldots, a_{j}\right] \times & d(\mu)\left[a_{j+1}, \ldots, a_{k}\right] \\
& \equiv d(\mu)\left[a_{1}, \ldots, a_{j}\right] \times d(\hat{\lambda})\left[a_{j+1}, \ldots, a_{k}\right] \bmod Z_{3} .
\end{aligned}
$$

For $k=2$ this is a classical result on parallelograms. The lemma was proved for $k=3$ by $\mathrm{H}$. Hadwiger [8] and for $k=3$ and $k=4$ by B. Jessen [12]. The following proof for arbitrary $k$ is due to A. Thorup.

Proof. For fixed $\lambda, \mu \in L$ and every pair $x_{1} \in \Pi\left(V_{1}\right), x_{2} \in \Pi\left(V_{2}\right)$, where $V=V_{1} \oplus V_{2}$, we denote by $x_{1} * x_{2}$ the element of $\Pi$ determined by

$$
x_{1} * x_{2}=d(\lambda) x_{1} \times d(\mu) x_{2}-d(\mu) x_{1} \times d(\hat{\lambda}) x_{2} .
$$

From Lemma 1 it follows that

$$
\begin{aligned}
(d(\hat{\lambda}+\mu)-d(\hat{\lambda})-d(\mu))\left[a_{1}, \ldots, a_{k}\right] \\
\quad=\sum_{j=1}^{k-1} d(\hat{\lambda})\left[a_{1}, \ldots, a_{j}\right] \times d(\mu)\left[a_{j+1}, \ldots, a_{k}\right] \\
=\sum_{j=1}^{k-1} d(\mu)\left[a_{1}, \ldots, a_{j}\right] \times d(\hat{\lambda})\left[a_{j+1}, \ldots, a_{k}\right] .
\end{aligned}
$$

Hence

$$
\sum_{j=1}^{k-1}\left[a_{1}, \ldots, a_{j}\right] *\left[a_{j+1}, \ldots, a_{k}\right]=0 .
$$


We must prove that all terms of this sum belong to $Z_{3}$. We do this by proving that if for some $i \in\{2, \ldots, k-1\}$ we have

$$
\sum_{j=1}^{i}\left[a_{1}, \ldots, a_{j}\right] *\left[a_{j+1}, \ldots, a_{k}\right] \in Z_{3}
$$

for arbitrary linearly independent vectors $a_{1}, \ldots, a_{k}$, then

$$
\left[b_{1}, \ldots, b_{i}\right] *\left[a_{i+1}, \ldots, a_{k}\right] \in Z_{3}
$$

for arbitrary linearly independent vectors $b_{1}, \ldots, b_{i}, a_{i+1}, \ldots, a_{k}$.

For arbitrary natural numbers $n_{1}>1, \ldots, n_{i-1}>1$ and for $j \in\{2, \ldots, i\}$ we introduce the abbreviations

$$
\begin{aligned}
& { }^{2}\left[a_{1}, \ldots, a_{j}\right]=\left[a_{1}, \ldots, a_{j}\right]-\left[a_{1}, a_{2} / n_{i-1}, \ldots, a_{j} / n_{i-1}\right] \\
& { }^{3}\left[a_{1}, \ldots, a_{j}\right]={ }^{2}\left[a_{1}, \ldots, a_{j}\right]-{ }^{2}\left[a_{1}, a_{2}, a_{3} / n_{i-2}, \ldots, a_{j} / n_{i-2}\right] \\
& \ldots \\
& { }^{j}\left[a_{1}, \ldots, a_{j}\right]={ }^{j-1}\left[a_{1}, \ldots, a_{j}\right]-{ }^{j-1}\left[a_{1}, \ldots, a_{j-1}, a_{j} / n_{i-j+1}\right] .
\end{aligned}
$$

The vector space spanned by $a_{j+1}, \ldots, a_{k}$ we denote by $V_{j}$.

We replace $a_{2}, \ldots, a_{k}$ in (1) by $a_{2} / n_{i-1}, \ldots, a_{k} / n_{i-1}$ and multiply by $n_{i-1}$. Since, by the corollary of Lemma 1 ,

$$
n_{i-1}^{*}\left[a_{j+1} / n_{i-1}, \ldots, a_{k} / n_{i-1}\right] \equiv\left[a_{j+1}, \ldots, a_{k}\right] \bmod Z_{2}\left(V_{j}\right),
$$

we obtain the relation

$$
\sum_{j=1}^{i}\left[a_{1}, a_{2} / n_{i-1}, \ldots, a_{j} / n_{i-1}\right] *\left[a_{j+1}, \ldots, a_{k}\right] \in Z_{3} .
$$

Subtracting this relation from (1) we obtain

$$
\sum_{j=2}^{i}{ }^{2}\left[a_{1}, \ldots, a_{j}\right] *\left[a_{j+1}, \ldots, a_{k}\right] \in Z_{3} .
$$

We replace $a_{3}, \ldots, a_{k}$ in (3) by $a_{3} / n_{i-2}, \ldots, a_{k} / n_{i-2}$ and multiply by $n_{i-2}$. Since

$$
n_{i-2}\left[a_{j+1} / n_{i-2}, \ldots, a_{k} / n_{i-2}\right] \equiv\left[a_{j+1}, \ldots, a_{k}\right] \bmod Z_{2}\left(V_{j}\right),
$$

we obtain the relation

$$
\sum_{j=2}^{i}{ }^{2}\left[a_{1}, a_{2}, a_{3} / n_{i-2}, \ldots, a_{j} / n_{i-2}\right] *\left[a_{j+1}, \ldots, a_{k}\right] \in Z_{3} .
$$

Subtracting this relation from (3) we obtain

$$
\sum_{j=3}^{i}\left[a_{1}, \ldots, a_{j}\right] *\left[a_{j+1}, \ldots, a_{k}\right] \in Z_{3} .
$$


Proceeding in this manner we obtain after $i-1$ steps the relation

$$
\left[a_{1}, \ldots, a_{i}\right] *\left[a_{i+1}, \ldots, a_{k}\right] \in Z_{3} .
$$

From (4) we shall now deduce (2). We consider a set of linearly independent vectors $b_{1}, \ldots, b_{i}, a_{i+1}, \ldots, a_{k}$, and denote by $W$ the subspace of $V$ spanned by $b_{1}, \ldots, b_{i}$, and by $B$ a subspace of $A$ of direction $W$. In (4) we let $a_{1}, \ldots, a_{i}$ be any set of linearly independent vectors in $W$.

For a polytope $Q$ in a proper subspace of $B$ and a point $p \in B$ not in this subspace we denote by $p(Q)$ the pyramid

$$
p(Q)=\{p+t \overrightarrow{p q} \mid t \in[0,1], q \in Q\}
$$

with base $Q$ and vertex $p$. By $p(n)(Q)$, where $n>1$ is a natural number, we denote the truncated pyramid

$$
p(n)(Q)=\{p+t \overrightarrow{p q} \mid t \in[1 / n, 1], q \in Q\} .
$$

A polytope $p(n)(Q)$ obtained in this way from $Q$ with some $p$ and some $n>1$ will be called a stump with base $Q$.

For given natural number $n_{1}>1, \ldots, n_{i-1}>1$, the element ${ }^{i}\left[a_{1}, \ldots, a_{i}\right]$ of $\Pi(W)$ is represented by a pyramid $P$ in $B$ obtained in the following way (see fig. 3). Choose a point $p_{i} \in B$ and consider the points $p_{i-1}, \ldots, p_{0}$ determined by $\overrightarrow{p_{i} p_{i-1}}=a_{1}, \ldots, \overrightarrow{p_{1} p_{0}}=a_{i}$. Let

$$
Q_{0}=\left\{p_{0}\right\}
$$

and form successively the stumps

$$
\begin{aligned}
& Q_{1}=p_{1}\left(n_{1}\right)\left(Q_{0}\right) \\
& Q_{2}=p_{2}\left(n_{2}\right)\left(Q_{1}\right) \\
& \ldots \\
& Q_{i-1}=p_{i-1}\left(n_{i-1}\right)\left(Q_{i-2}\right) .
\end{aligned}
$$

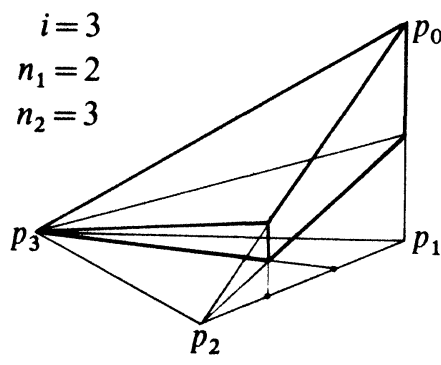

Fig. 3

Then the pyramid $P=p_{i}\left(Q_{i-1}\right)$ represents ${ }^{i}\left[a_{1}, \ldots, a_{i}\right]$.

A polytope $P$ in $B$ obtainable in this way from a point $p_{i}$, linearly independent vectors $a_{1}, \ldots, a_{i}$, and natural numbers $n_{1}>1, \ldots, n_{i-1}>1$, will be called a special pyramid in $B$. The relation (4) means that we have

$$
[P] *\left[a_{i+1}, \ldots, a_{k}\right] \in Z_{3}
$$

for every special pyramid $P$ in $B$. From this we deduce (2) by proving that any simplex in $B$ belongs to the class of polytopes obtained from the special pyramids by successive elementary subtractions.

A polytope as the $Q_{l}$ above obtained from a point $p_{l}$ in some $l$-dimensional subspace $C$ of $B$, linearly independent vectors $a_{i-1+1}, \ldots, a_{i}$ in $C$, and natural numbers $n_{1}>1, \ldots, n_{l}>1$, will be called a special stump in $C$. As a special 
pyramid is simply a pyramid whose base is a special stump, it suffices to prove, for any affine space $C$, that any simplex in $C$ belongs to the class of polytopes obtained from the special stumps by successive elementary subtractions.

As a special stump is a stump whose base is a special stump, an inductive argument shows that it is sufficient to prove that any simplex $S$ in $C$ belongs to the class $\mathfrak{D}$ of polytopes obtained by successive elementary subtractions from the stumps whose bases are simplices.

In a suitable coordinate system in $C$ with base vectors $c_{1}, \ldots, c_{l}$, the simplex $S$ is determined by

$$
S=\left\{\left(t_{1}, \ldots, t_{l}\right) \mid 0 \leqq t_{1} \leqq \ldots \leqq t_{1} \leqq 1\right\} .
$$

Consider now (see fig. 4) for $i=0,1, \ldots$ the polytopes

$$
\begin{aligned}
& S_{i}^{1}=\left\{\left(t_{1}, \ldots, t_{l}\right) \mid 0 \leqq t_{l} \leqq \ldots \leqq t_{1}, i \leqq t_{1} \leqq i+1\right\} \\
& S_{i}^{2}=\left\{\left(t_{1}, \ldots, t_{l}\right) \in S_{i+1}^{1} \mid t_{2} \geqq t_{1}-1\right\} \\
& S_{i}^{3}=\left\{\left(t_{1}, \ldots, t_{l}\right) \in S_{i+1}^{2} \mid t_{3} \geqq t_{2}-1\right\} \\
& \cdots \\
& S_{i}^{l}=\left\{\left(t_{1}, \ldots, t_{l}\right) \in S_{i+1}^{l-1} \mid t_{l} \geqq t_{l-1}-1\right\} .
\end{aligned}
$$

(We shall actually use only those $S_{i}^{j}$ for which $i+j \leqq l+1$ ) The polytopes $T_{i}$ $=S_{1}^{1} \cup \ldots \cup S_{i}^{1}, i \geqq 1$, are stumps whose bases are simplices; hence they belong to $\mathfrak{D}$. Since $T_{i}$ for $i \geqq 2$ is composed of $T_{i-1}$ and $S_{i}^{1}$, it follows that all $S_{i}^{1}, i \geqq 1$, belong to $\mathfrak{D}$. We want to show that also $S_{0}^{1}=S$ belongs to $\mathfrak{D}$. Let $\tau_{1}, \tau_{2}, \ldots, \tau_{l}$ denote the translations determined by $c_{1}, c_{1}+c_{2}, \ldots, c_{1}+\ldots+c_{l}$. Then for $i$ $\geqq 0$ we have decompositions

$$
\begin{aligned}
& S_{i+1}^{1}=\tau_{1} S_{i}^{1} \cup S_{i}^{2} \\
& S_{i+1}^{2}=\tau_{2} S_{i}^{2} \cup S_{i}^{3} \\
& \cdots \\
& S_{i+1}^{l-1}=\tau_{l-1} S_{i}^{l-1} \cup S_{i}^{l}
\end{aligned}
$$

and, moreover, we have the relation

$$
S_{i+1}^{l}=\tau_{l} S_{i}^{l} .
$$

Using the decompositions when $i \geqq 1$, we prove successively that all $S_{i}^{2}, S_{i}^{3}, \ldots, S_{i}^{l}, i \geqq 1$, belong to $\mathfrak{D}$. Using the relation when $i=0$, we see that $S_{0}^{l}$ belongs to $\mathfrak{D}$, and now, from the decompositions when $i=0$, we prove successively that $S_{0}^{l-1}, \ldots, S_{0}^{1}$ belong to $\mathcal{D}$. 

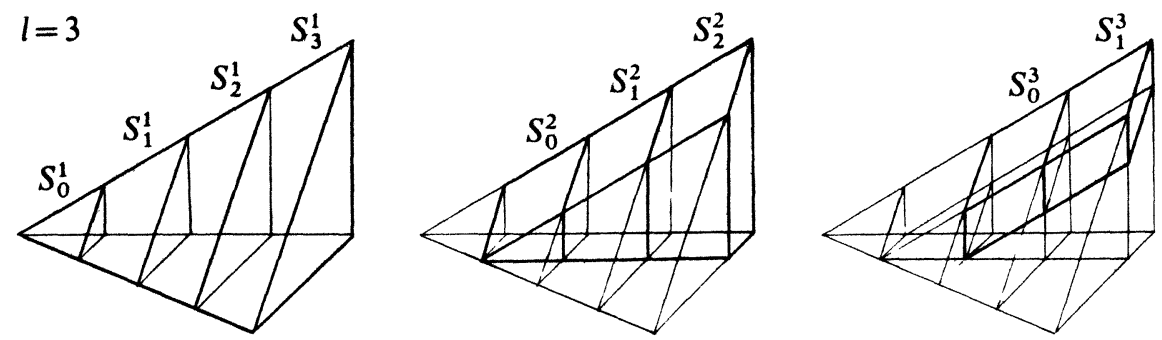

Fig. 4

\section{Polynomic functions.}

Our investigation will depend on certain purely algebraic theorems. It will be convenient to present these theorems in a somewhat more general form than that in which they are actually needed.

Let $L$ and $E$ be abelian groups with addition as composition. For every function $d: L \rightarrow E$ and every $\alpha \in L$ we define the function $\Delta_{\alpha} d: L \rightarrow E$ by

$$
\Delta_{\alpha} d(\hat{\lambda})=d(\hat{\lambda}+\alpha)-d(\hat{\lambda}) \text {. }
$$

For arbitrary $\alpha, \beta \in L$ we have $\Delta_{\beta} \Delta_{\alpha} d=\Delta_{\alpha} \Delta_{\beta} d$. For $\Delta_{\alpha_{p}} \ldots \Delta_{\alpha_{1}} d$, where $\alpha_{1}, \ldots, \alpha_{p}$ are all $=\alpha$, we write $\Delta_{\alpha}^{p} d$.

As a generalization of the notion of a polynomium we define polynomic functions from $L$ into $E$ as follows.

Let $k \geqq 0$ be an integer. A function $d: L \rightarrow E$ is called polynomic of degree $k$, if either $d$ is the zero function, or $\Delta_{\alpha_{k}} \ldots \Delta_{\alpha_{1}} d$ is constant for every set $\alpha_{1}, \ldots, \alpha_{k} \in L$, and this constant is not 0 for all sets $\alpha_{1}, \ldots, \alpha_{k}$. Thus the zero function is polynomic of degree $k$ for every $k$, whereas for a polynomic function which is not the zero function, the degree is well-determined. The polynomic functions of degree 0 are the constants, and the polynomic functions of degree $\leqq k$ are for every $k$ the functions for which $\Delta_{\alpha_{k}} \ldots \Delta_{\alpha_{1}} d$ is constant for every set $\alpha_{1}, \ldots, \alpha_{k} \in L$, or, equivalently, for which $\Delta_{\alpha_{k+1}} \ldots \Delta_{\alpha_{1}} d$ is the zero function for every set $\alpha_{1}, \ldots, \alpha_{k+1} \in L$.

For a polynomic function $d: \mathbf{Z} \rightarrow E$ of degree $\leqq k$ we have for every $q \in \mathbf{Z}$

$$
d(q)=\sum_{p=0}^{k}\left(\begin{array}{l}
q \\
p
\end{array}\right) \Delta_{1}^{p} d(0) .
$$

Consequently, for a polynomic function $d: L \rightarrow E$ of degree $\leqq k$ we have for every $\alpha \in L$ and every $q \in \mathbf{Z}$

$$
d(q \alpha)=\sum_{p=0}^{k}\left(\begin{array}{l}
q \\
p
\end{array}\right) \Delta_{\alpha}^{p} d(0) .
$$

Suppose now that $E$ is uniquely divisible, i.e., that $E$ is a vector space over $Q$. Let $k \geqq 1$. For an arbitrary multi-additive function $f: L^{k} \rightarrow E$ we define the symmetrized function $f^{\text {sym }}: L^{k} \rightarrow E$ by 


$$
f^{\mathrm{sym}}\left(i_{1}, \ldots, i_{k}\right)=\frac{1}{k !} \sum f\left(i_{\pi(1)}, \ldots, i_{\pi(k)}\right),
$$

where the summation is over all permutations $\pi$ of $\{1, \ldots, k\}$. It is also multiadditive. If $f$ is symmetric, we have $f^{\text {sym }}=f$. The function $d: L \rightarrow E$ defined by

$$
d(\hat{\lambda})=f(\hat{\lambda}, \ldots, \hat{\lambda})=f^{\mathrm{sym}}(\hat{\lambda}, \ldots, \hat{\lambda}),
$$

i.e., the function obtained by considering $f$ or $f^{\text {sym }}$ on the diagonal of $L^{k}$, is polynomic of degree $k$, since

$$
\Delta_{x_{k}} \ldots \Delta_{x_{1}} d(\lambda)=k ! f^{\text {sym }}\left(\alpha_{1}, \ldots, \alpha_{k}\right) .
$$

Such a function $d$ is called homogenic of degree $k$. Clearly, $d(0)=0$. One sees, that if $d$ is homogenic of degree $k$, then there exists just one symmetric, multiadditive function $d: L^{k} \rightarrow E$, such that $d(\hat{\lambda})=d(\lambda, \ldots, \hat{\lambda})$, namely the function determined by

$$
d\left(\alpha_{1}, \ldots, \alpha_{k}\right)=\frac{1}{k !} \Delta_{\alpha_{k}} \ldots \Delta_{\alpha_{1}} d(\hat{\lambda}) .
$$

Note that a homogenic function of degree 1 is simply an additive function $d: L \rightarrow E$. For such a function we have $d=d$.

A function $d: L \rightarrow E$ is called homogenic of degree 0 if it is a constant.

THEOREM A. Let $L$ be an abelian group and $E$ a uniquely divisible abelian group. Any polynomic function $d: L \rightarrow E$ of degree $\leqq k$ has a unique decomposition

$$
d=d_{0}+d_{1}+\ldots+d_{k},
$$

where $d_{i}: L \rightarrow E$ is homogenic of degree $i$. The components $d_{i}$ are determined recursively by the formulae

$$
\begin{aligned}
& d_{k}\left(\alpha_{1}, \ldots, \alpha_{k}\right)=\frac{1}{k !} \Delta_{\alpha_{k}} \ldots \Delta_{\alpha_{1}} d(\hat{\lambda}) \\
& d_{i}\left(\alpha_{1}, \ldots, \alpha_{i}\right)=\frac{1}{i !} \Delta_{\alpha_{i}} \ldots \Delta_{\alpha_{1}}\left(d-d_{k}-\ldots-d_{i+1}\right)(\hat{\lambda}) \quad \text { for } i<k .
\end{aligned}
$$

Proof. Since $d$ is polynomic of degree $\leqq k$, the function $\Delta_{\alpha_{k}} \ldots \Delta_{\alpha_{1}} d$ is constant for every set $\alpha_{1}, \ldots, \alpha_{k} \in L$. Let its value be denoted by $f\left(\alpha_{1}, \ldots, \alpha_{k}\right)$. The function $f: L^{k} \rightarrow E$ is clearly symmetric. It is also multi-additive. In order to prove this, it is enough to prove the additivity in the last variable. For given $\alpha_{1}, \ldots, \alpha_{k-1}$ we write $\Delta_{\alpha_{k-1}} \ldots \Delta_{\alpha_{1}} d(\lambda)=g(\lambda)$. Then we must prove that

$$
\Delta_{\alpha+\beta} g(\hat{\lambda})=\Delta_{\alpha} g(\hat{\lambda})+\Delta_{\beta} g(\hat{\lambda}),
$$

and this is clear, since $\Delta_{\alpha+\beta} g(\hat{\lambda})=\Delta_{\alpha} g(\hat{\lambda}+\beta)+\Delta_{\beta} g(\hat{\lambda})$, and $\Delta_{\alpha} g$ is a constant. 
We now put $d_{k}(\lambda)=(1 / k !) f(\lambda, \ldots, \lambda)$. Then $d_{k}$ is homogenic of degree $k$, and

$$
\tilde{d}_{k}\left(\alpha_{1}, \ldots, \alpha_{k}\right)=\frac{1}{k !} f\left(\alpha_{1}, \ldots, \alpha_{k}\right) .
$$

The function $d-d_{k}$ is therefore polynomic of degree $\leqq k-1$. The existence of the decomposition, and the recursion formulae, therefore follow by induction. Its uniqueness also follows by induction, since for any decomposition of the considered type we must have $\Delta_{\alpha_{k}} \ldots \Delta_{\alpha_{1}} d=\Delta_{\alpha_{k}} \ldots \Delta_{\alpha_{1}} d_{k}$, and hence $d_{k}(\lambda)$ $=(1 / k !) f(\lambda, \ldots, \lambda)$.

Assume now that $L$ and $E$ are commutative rings. The terms polynomic and homogenic will refer to the additive structures of $L$ and $E$. We shall be concerned with a special class of polynomic functions.

THEOREM B. Let $L$ be a commutative ring containing $\mathrm{Q}$, and $E$ a commutative ring containing $Z$. Let $d: L \rightarrow E$ be a function satisfying the following conditions:

(i) $d$ is multiplicative, and $d(0)=0, d(1)=1$;

(ii) $d$ is polynomic of degree $\leqq k$.

Then $E$ contains $Q$, i.e., every $n \in \mathbf{Z} \backslash\{0\}$, considered as an element of $E$, is invertible. More precisely, it has the inverse

$$
\frac{1}{n}=\sum_{p=1}^{k} \frac{k !}{p}\left(\begin{array}{c}
n k !-1 \\
p-1
\end{array}\right) \Delta_{1 / n k !}^{p} d(0) .
$$

The homogenic components $d_{0}, d_{1}, \ldots, d_{k}$ of $d$ are determined by $d_{0}=0$ and

$$
d_{i}(\lambda)=d(\lambda) \xi_{i} \quad \text { for } i \in\{1, \ldots, k\},
$$

where

$$
1=\xi_{1}+\ldots+\xi_{k}
$$

is a decomposition of $1 \in E$ into orthogonal idempotents $\xi_{i}=d_{i}(1)$.

Note that the first part of the theorem implies that $E$ (as additive group) is uniquely divisible, so that Theorem $A$ is applicable, and it makes sense to speak of the homogenic components of $d$. For the first part of the theorem, the multiplicativity of $d$ is not needed.

Proof. (a) Since $d(0)=0$, we have for every $\alpha \in L$ and every $q \in Z$

$$
d(q \alpha)=\sum_{p=1}^{k}\left(\begin{array}{l}
q \\
p
\end{array}\right) \Delta_{\alpha}^{p} d(0)
$$


For $q=n k$ !, where $n \in \mathbf{Z} \backslash\{0\}$, all the binomial coefficients are divisible by $n$. By choosing $\alpha=1 / n k$ ! we therefore find

$$
1=d(1)=n \sum_{p=1}^{k} c_{n, p} \Delta_{1 / n k}^{p} d(0)
$$

where the $c_{n, p}$ are the integers

$$
c_{n, p}=\frac{k !}{p}\left(\begin{array}{c}
n k !-1 \\
p-1
\end{array}\right) .
$$

(b) Since $d(0)=0$, we have $d_{0}=0$, so that the decomposition of $d$ into homogenic components has the form

$$
d=d_{1}+\ldots+d_{k} .
$$

Using the multiplicativity of $d$ we find

$$
\begin{aligned}
d(\hat{\lambda} \mu) & =d_{1}(\hat{\lambda} \mu)+\ldots+d_{k}(\hat{\lambda} \mu) \\
=d(\hat{\lambda}) d(\mu) & =d(\hat{\lambda}) d_{1}(\mu)+\ldots+d(\hat{\lambda}) d_{k}(\mu) .
\end{aligned}
$$

Here stand, for fixed $\lambda$, two decompositions of the polynomic function $\mu \mapsto d(\lambda \mu)$ into homogenic components. Consequently, $d_{i}(\lambda \mu)=d(\hat{\lambda}) d_{i}(\mu)$ for every $i \in\{1, \ldots, k\}$. Hence

$$
\begin{aligned}
d(\hat{\lambda}) d_{i}(\mu) & =d_{1}(\hat{\lambda}) d_{i}(\mu)+\ldots+d_{i}(\hat{\lambda}) d_{i}(\mu)+\ldots+d_{k}(\hat{\lambda}) d_{i}(\mu) \\
& =0+\ldots+d_{i}(\hat{\lambda} \mu)+\ldots+0 .
\end{aligned}
$$

Here stand, for fixed $\mu$, two decompositions of the polynomic function $\lambda \mapsto d(\hat{\lambda}) d_{i}(\mu)$ into homogenic components. Consequently

$$
\begin{aligned}
& d_{i}(\hat{\lambda}) d_{i}(\mu)=d_{i}(\hat{\lambda} \mu) \\
& d_{j}(\hat{\lambda}) d_{i}(\mu)=0 \quad \text { for } j \neq i .
\end{aligned}
$$

Thus, on placing $d_{i}(1)=\xi_{i}$, we have $\xi_{i}^{2}=\xi_{i}$, and $\xi_{j} \xi_{i}=0$ for $j \neq i$, so that $\xi_{1}, \ldots, \xi_{k}$ are orthogonal idempotents. Moreover, $1=d(1)=\xi_{1}+\ldots+\xi_{k}$. Finally, since $d_{i}(\hat{\lambda} \mu)=d(\hat{\lambda}) d_{i}(\mu)$, we have $d_{i}(\hat{\lambda})=d(\hat{\lambda}) \xi_{i}$.

Since $\xi_{1}, \ldots, \xi_{k}$ are orthogonal idempotents, the sets $\xi_{i} E$ will be orthogonal subrings of $E$. Note that the range of each $d_{i}$ belongs to $\xi_{i} E$, and that, consequently, also the range of each $d_{i}$ belongs to $\xi_{i} E$.

THEOREM C. Let $L$ be a commutative ring containing $Q$, and $E$ a commutative ring containing $\mathbf{Z}$.

If $\varphi: L \rightarrow E$ is a ring homomorphism with $\varphi(1)=1$, and if $1=\xi_{1}+\ldots+\xi_{k}$ is a 
decomposition of $1 \in E$ into orthogonal idempotents, then the function $d: L \rightarrow E$ defined by

$$
d(\lambda)=\varphi(\lambda) \xi_{1}+\ldots+\varphi\left(\lambda^{k}\right) \xi_{k}
$$

satisfies the conditions

(i) $d$ is multiplicative, and $d(0)=0, d(1)=1$;

(ii) $d$ is polynomic of degree $\leqq k$;

(iii) $\lambda_{i}\left(\lambda_{1}, \ldots, \lambda_{i}\right)=d_{i}\left(1, \ldots, 1, \lambda_{1} \ldots \lambda_{i}\right)$;

(iv) $\tilde{d}_{i}(1, \ldots, 1, \lambda \mu)=\tilde{d}_{i}(1, \ldots, 1, \lambda) \tilde{d}_{i}(1, \ldots, 1, \mu)$;

(v) $\lambda_{i}\left(\lambda_{1}, \ldots, \lambda_{i}\right)=\varphi\left(\hat{\lambda}_{1} \ldots \lambda_{i}\right) \xi_{i}$

(vi) $d_{i}(1, \ldots, 1)=\xi_{i}$;

(vii) $\mathfrak{d}_{1}(\lambda)+d_{2}(1, \lambda)+\ldots+d_{k}(1, \ldots, 1, \lambda)=\varphi(\lambda)$.

Conversely, if a function $d: L \rightarrow E$ satisfies the conditions (i), (ii), (iii), then there exist a unique ring homomorphism $\varphi: L \rightarrow E$ with $\varphi(1)=1$, and a unique decomposition $1=\xi_{1}+\ldots+\xi_{k}$ of $1 \in E$ into orthogonal idempotents, such that $d$ is determined by $\left({ }^{*}\right)$.

Note that, in the first part of the theorem, the existence of the homomorphism implies that $E$ contains $\mathrm{Q}$, and that, in the second part, by Theorem B, the conditions (i) and (ii) imply that $E$ contains $Q$. Note also that condition (iii) simply means that $\lambda_{i}\left(\lambda_{1}, \ldots, \lambda_{i}\right)$ depends on $\lambda_{1} \ldots \lambda_{i}$ only.

Proof. (a) Suppose that $d$ has the form $\left({ }^{*}\right)$.

Condition (i) is immediate. Conditions (ii) and (v) follow by observing that the function $\hat{\lambda} \mapsto \varphi\left(\lambda^{i}\right) \xi_{i}$ is the restriction to the diagonal of $L^{i}$ of the multiadditive, symmetric function $\left(\lambda_{1}, \ldots, \lambda_{i}\right) \mapsto \varphi\left(\lambda_{1} \ldots \lambda_{i}\right) \xi_{i}$. The remaining conditions (iii), (iv), (vi), (vii) are easy consequences.

(b) Suppose that $d$ satisfies (i), (ii), (iii).

Theorem B gives us the orthogonal idempotents $\xi_{1}, \ldots, \xi_{k}$ and the expressions $d_{i}(\lambda)=d(\lambda) \xi_{i}$ for the homogenic components.

Consider now for each $i$ the function $\varphi_{i}: L \rightarrow E$ determined by $\varphi_{i}(\lambda)$ $=\mathfrak{d}_{i}(1, \ldots, 1, \lambda)$. Clearly, it is additive. We shall prove that it is also multiplicative, i.e., that (iv) is satisfied. Using (iii) we find

$$
\varphi_{i}\left(\lambda^{i}\right)=d_{i}(\lambda, \ldots, \lambda)=d_{i}(\lambda)=d(\lambda) \xi_{i} .
$$

This shows that the function $\lambda \mapsto \varphi_{i}\left(\lambda^{i}\right)$ is multiplicative, i.e., that

$$
\varphi_{i}\left(\lambda^{i} \mu^{i}\right)=\varphi_{i}\left(\lambda^{i}\right) \varphi_{i}\left(\mu^{i}\right) .
$$

Thus, for fixed $\mu$, the two multi-additive, symmetric functions

$$
\left(\lambda_{1}, \ldots, \lambda_{i}\right) \mapsto \varphi_{i}\left(\lambda_{1} \ldots \lambda_{i} \mu^{i}\right) \quad \text { and } \quad\left(\lambda_{1}, \ldots, \lambda_{i}\right) \mapsto \varphi_{i}\left(\lambda_{1} \ldots \lambda_{i}\right) \varphi_{i}\left(\mu^{i}\right)
$$


coincide on the diagonal of $L^{i}$. Hence they are identical, i.e., we have $\varphi_{i}\left(i \mu^{i}\right)$ $=\varphi_{i}(\lambda) \varphi_{i}\left(\mu^{i}\right)$. Repeating the argument, we find $\varphi_{i}(\lambda \mu)=\varphi_{i}(i) \varphi_{i}(\mu)$.

We now consider the function $\varphi: L \rightarrow E$ defined by (vii), i.e., by

$$
\varphi(\lambda)=\varphi_{1}(\dot{\lambda})+\ldots+\varphi_{k}(\dot{\lambda}) .
$$

We find $\varphi(1)=d_{1}(1)+\ldots+d_{k}(1)=1$. Since each $\varphi_{i}$ is additive, $\varphi$ is additive; and since each $\varphi_{i}$ is multiplicative, and the terms are orthogonal, $\varphi$ is multiplicative. Hence $\varphi$ is a homomorphism with $\varphi(1)=1$.

Finally, since $\xi_{i}$ is orthogonal to the range of $\varphi_{j}$, when $j \neq i$, we find

$$
\varphi\left(\hat{\lambda}^{i}\right) \xi_{i}=\varphi_{i}\left(\hat{\lambda}^{i}\right) \xi_{i}=d(\hat{\lambda}) \xi_{i},
$$

whence $(*)$.

Note that if $L$ is a field, the ring homomorphism $\varphi: L \rightarrow E$ must be injective, so that $\varphi$ determines an embedding of $L$ in $E$ (an extension of the embedding of $Q$ ). In this case we may, for every $\lambda \in L$, use $\lambda$ also as notation for the corresponding element of $E$. The decomposition of $d$ thereby takes the form

$$
d(\hat{\lambda})=\lambda \xi_{1}+\ldots+i^{k} \xi_{k} .
$$

\section{The structure of the polytope group modulo translations.}

In our study of $\Pi=\Pi(V)$, the commutative subring $E$ of End $\Pi$ generated by the set $\{d(\hat{\lambda}) \mid \lambda \in L\}$ will play a decisive role. Since $d: L \rightarrow \operatorname{End} \Pi$ is multiplicative, $E$ consists of all endomorphisms

$$
\alpha_{v}=\sum_{\lambda \in L} v(\lambda) d(\lambda)
$$

where $v: L \rightarrow Z$ has finite support. The ring $E$ contains $Z$. We proceed in a number of steps.

(a) Let $a_{1}, \ldots, a_{k}$ be linearly independent vectors, and write

$$
x_{i j}=\left[a_{i+1}, \ldots, a_{j}\right] \quad \text { for } 0 \leqq i<j \leqq k .
$$

The formula of Lemma 1 (with $\alpha$ instead of $\mu$ ) can then be written

$$
\Delta_{\alpha} d(\lambda) x_{0 k}=\sum_{j=1}^{k} d(\alpha) x_{0 j} \times d(i) x_{j k} .
$$

(The term corresponding to $j=k$ means $d(\alpha) x_{0 k}$.) Hence, for arbitrary $\lambda, \alpha_{1}, \ldots, \alpha_{p} \in L$, where $0<p \leqq k$, we have

$$
\Delta_{\alpha_{p}} \ldots \Delta_{\alpha_{1}} d(\hat{\lambda}) x_{0 k}=\sum_{0<i_{1}<\ldots<i_{p} \leqq k} d\left(\alpha_{1}\right) x_{0 i_{1}} \times \ldots \times d\left(\alpha_{p}\right) x_{i_{p-1} i_{p}} \dot{x} d(\dot{\lambda}) x_{i_{p} k} .
$$


(A term corresponding to indices $i_{1}, \ldots, i_{p}$, where $i_{p}=k$, means $d\left(\alpha_{1}\right) x_{0 i_{1}} \times \ldots$ $\left.\times d\left(\alpha_{p}\right) x_{i_{p-1} k}\right)$ In particular

$$
\Delta_{\alpha_{k}} \ldots \Delta_{\alpha_{1}} d(\hat{\lambda}) x_{0 k}=d\left(\alpha_{1}\right)\left[a_{1}\right] \times \ldots \times d\left(\alpha_{k}\right)\left[a_{k}\right] .
$$

Using that the elements $\left[a_{1}, \ldots, a_{k}\right]$ generate $\Pi$, and that

$$
d\left(\alpha_{1}\right)\left[a_{1}\right] \times \ldots \times d\left(\alpha_{k}\right)\left[a_{k}\right] \neq 0 \quad \text { when } \alpha_{1} \ldots \alpha_{k} \neq 0,
$$

we find:

The dilation operator $d: L \rightarrow E$ is polynomic of degree $k$.

For arbitrary $\lambda, \alpha_{1}, \ldots, \alpha_{p}$, where $0<p \leqq k$, the endomorphism $\Delta_{\alpha_{p}} \ldots \Delta_{\alpha_{1}} d(\lambda)$ maps $\Pi$ into $Z_{p}$.

(b) Thus the dilation operator $d: L \rightarrow E$ satisfies the conditions of Theorem B. Consequently, $E$ contains $\mathrm{Q}$, which implies that $\Pi$ is uniquely divisible, and we have the decomposition $d=d_{1}+\ldots+d_{k}$ of $d$ into homogenic components determined by $d_{i}(\hat{\lambda})=d(\hat{\lambda}) \xi_{i}$, where $1=\xi_{1}+\ldots+\xi_{k}$ is a decomposition of $1 \in E$ into orthogonal idempotents $\xi_{i}=d_{i}(1)$. Since $d$ is of degree $k$, we have $\xi_{k} \neq 0$.

Introducing the subgroups $\xi_{i} \Pi=\Xi_{i}$, we have

$$
\Pi=\Xi_{1} \oplus \ldots \oplus \Xi_{k} .
$$

Each of the subgroups $\Xi_{i}$ is stable under the automorphism $\bar{\varphi}$ for every automorphism $\varphi$ of $V$, since $\bar{\varphi} \xi_{i}=\xi_{i} \bar{\varphi}$. Instead of $\Xi_{i}$ we also write $\Xi_{i}(A)$ or $\Xi_{i}(V)$.

The notation $d_{i}(\hat{\lambda})$ for the homogenic components of the dilation operator, and the notation $d_{i}(1)=\xi_{i}$, will also be used in relation to the polytope groups modulo translations belonging to subspaces of $A$.

(c) Let $V=V_{1} \oplus \ldots \oplus V_{i}$, and let $x_{1} \in \Pi\left(V_{1}\right), \ldots, x_{i} \in \Pi\left(V_{i}\right)$. The function $\hat{i} \mapsto d(\hat{\lambda})\left(x_{1} \times \ldots \times x_{i}\right)$ from $L$ into the uniquely divisible group $\Pi$ is a polynomic function of degree $\leqq k$ with the homogenic components $\lambda \mapsto$ $d_{i}(\hat{\lambda})\left(x_{1} \times \ldots \times x_{i}\right)$. Since

$$
d(\hat{\lambda})\left(x_{1} \times \ldots \times x_{i}\right)=d(\hat{\lambda}) x_{1} \times \ldots \times d(\hat{\lambda}) x_{i}
$$

we have

$$
d(\lambda)\left(x_{1} \times \ldots \times x_{i}\right)=\sum_{j=i}^{k} \sum_{l_{1}+\ldots+l_{1}=j} d_{l_{1}}(\lambda) x_{1} \times \ldots \times d_{l_{i}}(\hat{\lambda}) x_{i} .
$$

Each term

$$
d_{l_{1}}(\lambda) x_{1} \times \ldots \times d_{l_{i}}(\hat{\lambda}) x_{i}=d_{l_{1}}(\hat{\lambda}, \ldots, \hat{\lambda}) x_{1} \times \ldots \times d_{l_{i}}(\hat{\lambda}, \ldots, \hat{\lambda}) x_{i}
$$

with $l_{1}+\ldots+l_{i}=j$ is the restriction of a multi-additive function from $L^{j}$ into $\Pi$ to the diagonal of $L^{j}$, namely the function obtained by replacing the $\hat{i}$ 's on 
the right hand side by $\lambda_{1}, \ldots, \lambda_{j}$, and is therefore homogenic of degree $j$. Hence we have

$$
\begin{aligned}
& d_{j}(\lambda)\left(x_{1} \times \ldots \times x_{i}\right)=0 \quad \text { for } j<i \\
& d_{j}(\lambda)\left(x_{1} \times \ldots \times x_{i}\right)=\sum_{l_{1}+\ldots+l_{i}=j} d_{l_{1}}(\hat{\lambda}) x_{1} \times \ldots \times d_{l_{i}}(\hat{\lambda}) x_{i} \quad \text { for } j \geqq i,
\end{aligned}
$$

in particular

$$
d_{i}(\hat{\lambda})\left(x_{1} \times \ldots \times x_{i}\right)=d_{1}(\lambda) x_{1} \times \ldots \times d_{1}(\hat{\lambda}) x_{i} .
$$

On placing $\lambda=1$, and considering only values $j \leqq i$, we obtain

$$
\begin{aligned}
& \xi_{j}\left(x_{1} \times \ldots \times x_{i}\right)=0 \quad \text { for } j<i \\
& \xi_{i}\left(x_{1} \times \ldots \times x_{i}\right)=-\overrightarrow{\xi_{1}} x_{1} \times \ldots \times \xi_{1} x_{i} .
\end{aligned}
$$

(d) Since $Z_{i}$ is generated by the elements $x_{1} \times \ldots \times x_{i}$, where $x_{1} \in$ $\Pi\left(V_{1}\right), \ldots, x_{i} \in \Pi\left(V_{i}\right)$, for all choices of $V_{1}, \ldots, V_{i}$ such that $V=V_{1} \oplus \ldots \oplus V_{i}$, the relation $\xi_{j}\left(x_{1} \times \ldots \times x_{i}\right)=0$ for $j<i$ implies that $Z_{i} \subseteq \Xi_{i} \oplus \ldots \oplus \Xi_{k}$. On the other hand, since $d_{i}(\lambda)=d(\lambda) \xi_{i}$, we have (for every $i$ )

$$
\lambda_{i}\left(\alpha_{1}, \ldots, \alpha_{i}\right)=\frac{1}{i !} \Delta_{\alpha_{1}} \ldots \Delta_{\alpha_{1}} d(\hat{\lambda}) \xi_{i},
$$

and consequently

$$
\xi_{i}=\Delta_{1}^{i} d(\hat{\lambda}) \frac{1}{i !} \xi_{i}
$$

From this it follows, since $\Delta_{1}^{i} d(\lambda)$, as shown in (a), maps $\Pi$ into $Z_{i}$, that $\Xi_{i} \leqq Z_{i}$, and hence that $\Xi_{i} \oplus \ldots \oplus \Xi_{k} \cong Z_{i}$. Thus we have

$$
Z_{i}=\Xi_{i} \oplus \ldots \oplus \Xi_{k} \text {. }
$$

(e) From $\Xi_{i} \subseteq Z_{i}$ and $\xi_{i} \Pi=\Xi_{i}=\xi_{i} \Xi_{i}$ it follows that $\Xi_{i}=\xi_{i} Z_{i}$. Since $Z_{i}$ is generated by the elements $x_{1} \times \ldots \times x_{i}$, where $x_{1} \in \Pi\left(V_{1}\right), \ldots, x_{i} \in \Pi\left(V_{i}\right)$, for all choices of $V_{1}, \ldots, V_{i}$ such that $V=V_{1} \oplus \ldots \oplus V_{i}$, it follows that $\Xi_{i}$ is generated by the elements $\xi_{i}\left(x_{1} \times \ldots \times x_{i}\right)$. Since $\xi_{i}\left(x_{1} \times \ldots \times x_{i}\right)=\xi_{1} x_{1} \times \ldots$ $\times \xi_{1} x_{i}$, we find:

The group $\Xi_{i}$ is generated by the elements $x_{1} \times \ldots \times x_{i}$, where $x_{1} \in \Xi_{1}\left(V_{1}\right), \ldots, x_{i} \in \Xi_{1}\left(V_{i}\right)$, for all choices of $V_{1}, \ldots, V_{i}$ such that $V$ $=V_{1} \oplus \ldots \oplus V_{i}$.

The subset of $\Xi_{i}$ consisting of these elements will be denoted by $\Gamma_{i}$. Note that $\Gamma_{1}=\Xi_{1}$. The whole group $\Pi$ is generated by the set $\Gamma=\Gamma_{1} \cup \ldots \cup \Gamma_{k}$. 
(f) Let $V=V_{1} \oplus V_{2}$. From Lemma 2 it follows that for arbitrary $x_{1} \in \Pi\left(V_{1}\right)$ and $x_{2} \in \Pi\left(V_{2}\right)$, and for arbitrary $i, \mu \in L$, we have

$$
d(\dot{\lambda}) x_{1} \times d(\mu) x_{2}-d(\mu) x_{1} \times d(\dot{\lambda}) x_{2} \in Z_{3} .
$$

Suppose now that $x_{1} \in \Xi_{1}\left(V_{1}\right)$ and $x_{2} \in \Xi_{1}\left(V_{2}\right)$. Then also

$$
\begin{aligned}
& d(\hat{\lambda}) x_{1} \in \Xi_{1}\left(V_{1}\right), d(\mu) x_{1} \in \Xi_{1}\left(V_{1}\right) \\
& d(\dot{\lambda}) x_{2} \in \Xi_{1}\left(V_{2}\right), d(\mu) x_{2} \in \Xi_{1}\left(V_{2}\right) .
\end{aligned}
$$

Hence, by (c),

$$
d(\hat{\lambda}) x_{1} \times d(\mu) x_{2} \in \Xi_{2}, \quad d(\mu) x_{1} \times d(\hat{\lambda}) x_{2} \in \Xi_{2},
$$

and consequently

$$
d(\hat{\lambda}) x_{1} \times d(\mu) x_{2}-d(\mu) x_{1} \times d(\hat{\lambda}) x_{2} \in \Xi_{2} .
$$

Since $Z_{3} \cap \Xi_{2}=\{0\}$, we conclude that

$$
d(\hat{\lambda}) x_{1} \times d(\mu) x_{2}=d(\mu) x_{1} \times d(\hat{\lambda}) x_{2} .
$$

In particular,

$$
d(\hat{\lambda}) x_{1} \times x_{2}=x_{1} \times d(\hat{\lambda}) x_{2} .
$$

By repeated use of this, and using the multiplicativity of $d$, we find:

If $V=V_{1} \oplus \ldots \oplus V_{i}$, and $x_{1} \in \Xi_{1}\left(V_{1}\right), \ldots, x_{i} \in \Xi_{1}\left(V_{i}\right)$, then for arbitrary $\hat{\lambda}_{1}, \ldots, \hat{\lambda}_{i} \in L$ we have

$$
d\left(\hat{\lambda}_{1}\right) x_{1} \times \ldots \times d\left(\hat{\lambda}_{i}\right) x_{i}=x_{1} \times \ldots \times x_{i-1} \times d\left(\hat{\lambda}_{1} \ldots \hat{\lambda}_{i}\right) x_{i} .
$$

In other words: For an element $x_{1} \times \ldots \times x_{i}$ of the generating set $\Gamma_{i}$ of $\Xi_{i}$, the element $d\left(\hat{\lambda}_{1}\right) x_{1} \times \ldots \times d\left(\lambda_{i}\right) x_{i}$ depends only on the product $\hat{\lambda}_{1} \ldots \hat{\lambda}_{i}$. An operator $d(\lambda)$ can be moved freely from one factor of $x_{1} \times \ldots \times x_{i}$ to another.

(g) Let $V=V_{1} \oplus \ldots \oplus V_{i}$, and let $x_{1} \in \Pi\left(V_{1}\right), \ldots, x_{i} \in \Pi\left(V_{i}\right)$. For the $i$ th homogenic component $\hat{i} \mapsto d_{i}(\lambda)\left(x_{1} \times \ldots \times x_{i}\right)$ of the function $i \mapsto$ $d(\lambda)\left(x_{1} \times \ldots \times x_{i}\right)$ from $L$ into $\Pi$ we found in (c) the expression

$$
d_{i}(\hat{\lambda})\left(x_{1} \times \ldots \times x_{i}\right)=d_{1}(\hat{\lambda}) x_{1} \times \ldots \times d_{1}(\hat{\lambda}) x_{i} .
$$

From this we deduce, for arbitrary $\lambda_{1}, \ldots, \lambda_{i} \in L$,

$$
\lambda_{i}\left(\hat{\lambda}_{1}, \ldots, \hat{\lambda}_{i}\right)\left(x_{1} \times \ldots \times x_{i}\right)=\frac{1}{i !} \sum d_{1}\left(\hat{\lambda}_{\pi(1)}\right) x_{1} \times \ldots \times d_{1}\left(\hat{\lambda}_{\pi(i)}\right) x_{i},
$$

where the summation is over all permutations $\pi$ of $\{1, \ldots, i\}$. Indeed, on both sides we have a symmetric, multi-additive function from $L^{i}$ into $\Pi$, whose restriction to the diagonal of $L^{i}$ is $d_{i}(\lambda)\left(x_{1} \times \ldots \times x_{i}\right)$. 
Suppose now, in particular, that $x_{1} \in \Xi_{1}\left(V_{1}\right), \ldots, x_{i} \in \Xi_{1}\left(V_{i}\right)$. Then the formula can be written

$$
d_{i}\left(\lambda_{1}, \ldots, \lambda_{i}\right)\left(x_{1} \times \ldots \times x_{i}\right)=\frac{1}{i !} \sum d\left(\lambda_{\pi(1)}\right) x_{1} \times \ldots \times d\left(\lambda_{\pi(i)}\right) x_{i} .
$$

For each term on the right hand side we have, by the result in (f),

$$
d\left(\hat{\lambda}_{\pi(1)}\right) x_{1} \times \ldots \times d\left(\lambda_{\pi(i)}\right) x_{i}=x_{1} \times \ldots \times x_{i-1} \times d\left(\lambda_{1} \ldots \lambda_{i}\right) x_{i} .
$$

Hence we find

$$
d_{i}\left(\lambda_{1}, \ldots, \lambda_{i}\right)\left(x_{1} \times \ldots \times x_{i}\right)=x_{1} \times \ldots \times x_{i-1} \times d\left(\lambda_{1} \ldots \lambda_{i}\right) x_{i},
$$

whence

$$
d_{i}\left(\hat{\lambda}_{1}, \ldots, \hat{\lambda}_{i}\right)\left(x_{1} \times \ldots \times x_{i}\right)=d_{i}\left(1, \ldots, 1, \lambda_{1} \ldots \hat{\lambda}_{i}\right)\left(x_{1} \times \ldots \times x_{i}\right) .
$$

Using that $\lambda_{i}\left(\lambda_{1}, \ldots, \lambda_{i}\right)$ vanishes on $\Xi_{j}$ for $j \neq i$ and all $\lambda_{1}, \ldots, \lambda_{i} \in L$ we see that

$$
\lambda_{i}\left(\lambda_{1}, \ldots, \lambda_{i}\right) x=d_{i}\left(1, \ldots, 1, \lambda_{1} \ldots \hat{\lambda}_{i}\right) x
$$

for all elements $x$ of the generating set $\Gamma=\Gamma_{1} \cup \ldots \cup \Gamma_{k}$ of $\Pi$, and hence for all $x \in \Pi$. Thus

$$
\lambda_{i}\left(\lambda_{1}, \ldots, \hat{\lambda}_{i}\right)=\lambda_{i}\left(1, \ldots, 1, \lambda_{1} \ldots \hat{\lambda}_{i}\right) .
$$

(h) Thus the dilation operator $d: L \rightarrow E$ satisfies not only the conditions (i) and (ii), but also the condition (iii) of Theorem $\mathrm{C}$. Consequently, the function $\varphi: L \rightarrow E$ determined by

$$
\varphi(\lambda)=d_{1}(\lambda)+d_{2}(1, \lambda)+\ldots+d_{k}(1, \ldots, 1, \lambda)
$$

is a ring homomorphism with $\varphi(1)=1$, and the decomposition of $d$ into homogenic components is determined by

$$
d(\lambda)=\varphi(\lambda) \xi_{1}+\ldots+\varphi\left(\lambda^{k}\right) \xi_{k} .
$$

Since $L$ is a field, $\varphi$ determines an embedding of $L$ into $E$ (extending the embedding of Q). For the element $\varphi(\lambda)$ of $E$ we shall therefore write $\lambda$, so that the preceding formulae take the form

$$
\lambda=\partial_{1}(\lambda)+d_{2}(1, \lambda)+\ldots+d_{k}(1, \ldots, 1, \lambda)
$$

and

$$
d(\lambda)=\lambda \xi_{1}+\ldots+\lambda^{k} \xi_{k} .
$$

Through the embedding of $L$ in $E$, the group $\Pi$ becomes a vector space over $L$, the scalar multiplication by an element $\lambda \in L$ being the endomorphism $\lambda$. The subgroups $Z_{i}$ and $\Xi_{i}$ become subspaces, and the expression for $d$ shows that the 
restriction of $d(\hat{i})$ to $\Xi_{i}$ is scalar multiplication by $\lambda_{i}^{i}$. The expression is called the spectral decomposition of $d$.

For an element $x_{1} \times \ldots \times x_{i}$ of the generating set $\Gamma_{i}$ of $\Xi_{i}$ we find from $(\mathrm{g})$ $\lambda\left(x_{1} \times \ldots \times x_{i}\right)=d_{i}(1, \ldots, 1, \hat{\lambda})\left(x_{1} \times \ldots \times x_{i}\right)=x_{1} \times \ldots \times x_{i-1} \times d(\hat{\lambda}) x_{i}$.

(j) Let $h: \Pi \rightarrow L$ be an additive, function, which is homogeneous with respect to $d(\hat{\lambda})$ of degree $i \in \mathrm{N}$, i.e., for which $h(d(\hat{\lambda}) x)=\hat{\lambda}^{i} h(x)$ for all $x \in \Pi$ and all $\lambda \in L$, and which is not the zero function. We then have

$$
\hat{\lambda}^{i} h(x)=h\left(\hat{\lambda} \xi_{1} x\right)+\ldots+h\left(\hat{\lambda}^{k} \xi_{k} x\right) .
$$

For a fixed $x$, the function $\hat{\lambda} \mapsto \hat{\lambda}^{i} h(x)$ from $L$ into $L$ is the restriction of the symmetric, multi-additive function $\left(\hat{\lambda}_{1}, \ldots, \hat{\lambda}_{i}\right) \mapsto \hat{\lambda}_{1} \ldots \hat{\lambda}_{i} h(x)$ to the diagonal of $L^{i}$, and the $j$ th term on the right hand side is the restriction of the symmetric, multi-additive function $\left(\hat{\lambda}_{1}, \ldots, \hat{\lambda}_{j}\right) \mapsto h\left(\hat{\lambda}_{1} \ldots \hat{\lambda}_{j} \xi_{j} x\right)$ to the diagonal of $L^{j}$. Choosing an $x$, for which $h(x) \neq 0$; we see that $i$ must be one of the numbers $1, \ldots, k$. Thereafter we see that for every $x$ and for arbitrary $i$ 's we must have

$$
\begin{aligned}
& h\left(\hat{\lambda}_{1} \ldots \hat{\lambda}_{j} \xi_{j} x\right)=0 \quad \text { for } j \neq i \\
& h\left(\hat{\lambda}_{1} \ldots \hat{\lambda}_{i} \xi_{i} x\right)=\lambda_{1} \ldots \hat{\lambda}_{i} h(x) .
\end{aligned}
$$

From this it immediately follows, that $h$ is a linear function on the vector space $\Pi$, which vanishes on $\Xi_{j}$ for $j \neq i$.

There is an obvious converse: If $h: \Pi \rightarrow L$ is a linear function which vanishes on $\Xi_{j}$ for $j \neq i$, then $h$ is homogeneous of degree $i$ with respect to $d(\hat{i})$.

The Hadwiger function $H_{\Phi}: \Pi \rightarrow L$ for an $i$-flag $\Phi$ is additive, and homogeneous of degree $i$ with respect to $d(\hat{\lambda})$, and is not the zero function. Hence it is a linear function which vanishes on $\Xi_{j}$ for $j \neq i$. Hence $\Xi_{i} \neq\{0\}$, that is to say, $\xi_{i} \neq 0$ for all $i \in\{1, \ldots, k\}$.

(k) From the spectral decomposition of $d$ it follows that, for an arbitrary function $v: L \rightarrow Z$ with finite support, the corresponding element $\alpha_{v}$ of the ring $E$ will be determined by

$$
\alpha_{v}=\sum_{\lambda \in L} v(\hat{\lambda}) d(\hat{\lambda})=\hat{\lambda}_{1} \xi_{1}+\ldots+\hat{\lambda}_{k} \xi_{k}
$$

where

$$
\left(i_{1}, \ldots, \lambda_{k}\right)=\sum_{\lambda \in L} v(\lambda)\left(\lambda_{,} \ldots, \lambda^{k}\right) .
$$

Hence $E$ consists of all $\alpha=\hat{\lambda}_{1} \xi_{1}+\ldots+\hat{\lambda}_{k} \xi_{k}$, where $\hat{\lambda}_{1}, \ldots, \hat{\lambda}_{k} \in L$. The endomorphism $\alpha$ is simply the linear transformation of the vector space $\Pi$ whose restriction to each $\Xi_{i}$ is scalar multiplication by $\lambda_{i}$. Since all $\Xi_{i}$ are $\neq\{0\}$, the coefficients $\hat{\lambda}_{1}, \ldots, \hat{\lambda}_{k}$ are uniquely determined by $\alpha$. Since 


$$
\begin{gathered}
\left(\hat{\lambda}_{1} \xi_{1}+\ldots+\lambda_{k} \xi_{k}\right)+\left(\mu_{1} \xi_{1}+\ldots+\mu_{k} \xi_{k}\right)=\left(\lambda_{1}+\mu_{1}\right) \xi_{1}+\ldots+\left(\lambda_{k}+\mu_{k}\right) \xi_{k} \\
\left(\lambda_{1} \xi_{1}+\ldots+\lambda_{k} \xi_{k}\right)\left(\mu_{1} \xi_{1}+\ldots+\mu_{k} \xi_{k}\right)=\lambda_{1} \mu_{1} \xi_{1}+\ldots+\lambda_{k} \mu_{k} \xi_{k}
\end{gathered}
$$

we see that $E$ is isomorphic to the product ring $L^{k}$, the isomorphism from $L^{k}$ onto $E$ being determined by $\left(\hat{\lambda}_{1}, \ldots, \hat{\lambda}_{k}\right) \mapsto \hat{\lambda}_{1} \xi_{1}+\ldots+\lambda_{k} \xi_{k}$.

From the expression for $\alpha_{v}$ in the form $\lambda_{1} \xi_{1}+\ldots+\lambda_{k} \xi_{k}$ it follows that $L^{k}$ as additive group is generated by the set $\left\{\left(\lambda_{,}, \ldots, \dot{i}^{k}\right) \mid i \in L\right\}$, and that $\alpha_{v}=0$, if and only if the function $v: L \rightarrow \mathrm{Z}$ satisfies the condition

$$
\sum_{i \in L} v\left(\lambda_{i}\right)\left(\lambda, \ldots, \hat{i}^{k}\right)=(0, \ldots, 0) .
$$

Examples of such relations were first given by H. Hadwiger [5].

Collecting the principal results of the preceding analysis, we see that we have proved the following theorem.

THEOREM 3. Let $E$ denote the subring of the endomorphism ring of the group $\Pi$ $=\Pi(V)$ which is generated by the set $\{d(\lambda) \mid \lambda \in L\}$. The function $d: L \rightarrow E$ is polynomic of degree $k$. The elements $\xi_{i}=d_{i}(1)=d_{i}(1, \ldots, 1)$ determine $a$ decomposition $1=\xi_{1}+\ldots+\xi_{k}$ of $1 \in E$ into orthogonal idempotents $\xi_{i} \neq 0$, and thereby a decomposition

$$
\Pi=\Xi_{1} \oplus \ldots \oplus \Xi_{k}
$$

of the group $\Pi$ into the groups $\Xi_{i}=\xi_{i} \Pi$. For every $i$ we have

$$
Z_{i}=\Xi_{i} \oplus \ldots \oplus \Xi_{k} .
$$

The map $\hat{\lambda} \mapsto d_{1}(\hat{\lambda})+d_{2}(1, \hat{\lambda})+\ldots+\lambda_{k}(1, \ldots, 1, \hat{\lambda})$ determines an embedding of $L$ into $E$, and thereby makes the group $\Pi$ into a vector space over $L$, scalar multiplication by an element $\hat{\lambda} \in L$ being the endomorphism $\lambda_{\text {. The subgroups } Z_{i}}$ and $\Xi_{i}$ become subspaces. The group $\Xi_{i}$ is generated by the products $x_{1} \times \ldots \times x_{i}$, where $x_{1} \in \Xi_{1}\left(V_{1}\right) \ldots, x_{i} \in \Xi_{1}\left(V_{i}\right)$ and $V=V_{1} \oplus \ldots \oplus V_{i}$. For such a product the scalar multiplication by $\lambda$ is effected by applying $d(\hat{\lambda})$ to one of its factors.

The ring $E$ consists of all $\hat{\lambda}_{1} \xi_{1}+\ldots+\hat{\lambda}_{k} \xi_{k}$, where $\lambda_{1}, \ldots, \hat{\lambda}_{k} \in L$, and is isomorphic to the product ring $L^{k}$.

The dilation operator has the spectral decomposition

$$
d(\hat{\lambda})=\hat{\lambda} \xi_{1}+\ldots+\hat{\lambda}^{k} \xi_{k} .
$$

Our formulae for $\xi_{1} \ldots \ldots \xi_{k}$. and for $\lambda$ (as element of $E$ ). show us how to express any element $\lambda_{1} \xi_{1}+\ldots+\lambda_{k} \xi_{k}$ of $E$ by means of dilations, but they lead to complicated expressions. One reason for this is that the factors $1 / i$ ! in the expressions for the homogenic components in Theorem A must be determined by means of the formula in Theorem B. It is simpler to proceed as follows. 
Insert in the spectral decomposition of $d(\lambda)$ for $\lambda$ the $k$ values $i / k !, i \in\{1, \ldots, k\}$. By solving the equations we find expressions

$$
\frac{1}{i !} \xi_{i}=a_{i 1} d\left(\frac{1}{k !}\right)+\ldots+a_{i k} d\left(\frac{k}{k !}\right),
$$

where the coefficients $a_{i j}$ are integers. On the other hand, from $\lambda^{i} \xi_{i}=d(\lambda) \xi_{i}$ we find

$$
\dot{\lambda}_{i} \xi_{i}=\Delta_{1}^{i-1} \Delta_{\lambda_{i}} d(0) \frac{1}{i !} \xi_{i} .
$$

Thus we obtain an expression for $\lambda_{1} \xi_{1}+\ldots+\lambda_{k} \xi_{k}$ by means of dilations without having to use the formula in Theorem $B$ to get rid of the fractions. - For $k=2$ we find from

$$
\left.\begin{array}{l}
d\left(\frac{1}{2}\right)=\frac{1}{2} \xi_{1}+\frac{1}{4} \xi_{2} \\
d(1)=\xi_{1}+\xi_{2}
\end{array}\right\} \text { that }\left\{\begin{aligned}
\xi_{1} & =4 d\left(\frac{1}{2}\right)-d(1) \\
\frac{1}{2} \xi_{2} & =-2 d\left(\frac{1}{2}\right)+d(1)
\end{aligned}\right.
$$

whence

$$
\begin{aligned}
\hat{\lambda}_{1} \xi_{1}+\hat{\lambda}_{2} \xi_{2} & =4 d\left(\frac{1}{2} \hat{\lambda}_{1}\right)-d\left(\hat{\lambda}_{1}\right) \\
- & 2 d\left(\frac{1}{2} \hat{\lambda}_{2}+\frac{1}{2}\right)+2 d\left(\frac{1}{2} \hat{\lambda}_{2}\right)+2 d\left(\frac{1}{2}\right)+d\left(\hat{i}_{2}+1\right)-d\left(\hat{\lambda}_{2}\right)-d(1) .
\end{aligned}
$$

Another method is to develop formulae for expressing an arbitrary $x \in \Pi$ in terms of elements of the generating set $\Gamma=\Gamma_{1} \cup \ldots \cup \Gamma_{k}$ introduced in (e). It is enough to do this for elements $x$ which are represented by simplices. Such formulae can be found by induction after the dimension. For $\hat{\lambda}_{i}=1$, the above formula for $\hat{\lambda}_{i} \xi_{i}$ takes the form

$$
\xi_{i}=\frac{1}{i !} \xi_{i} \varepsilon_{i}, \quad \text { where } \varepsilon_{i}=\Delta_{1}^{i} d(0) .
$$

For the equivalence class $x=\left[a_{1}, \ldots, a_{k}\right]$ of a simplex, we have, using the notation from (a),

$$
\varepsilon_{i} x=\sum_{0<j_{1}<\ldots<j_{i-1}<k} x_{0 j_{1}} \times \ldots \times x_{j_{i-1} k} .
$$

Using (c) and (h) we therefore find

$$
\xi_{i} x=\sum_{0<j_{1}<\ldots<j_{i-1}<k} \xi_{1} x_{0 j_{1}} \times \ldots \times \xi_{1} x_{j_{1-2} j_{i-1}} \times d\left(\frac{1}{i !}\right) \xi_{1} x_{j_{i-1} k} .
$$

(In each term, the dilation $d(1 / i$ !) can be placed on any of the factors.)

For $k=1$ we have $\xi_{1}=1$. Suppose now that we have developed formulae for $\xi_{1}\left[a_{1}, \ldots, a_{j}\right]$ for all dimensions $j \in\{1, \ldots, k-1\}$. Then the expression 
for $\xi_{i}\left[a_{1}, \ldots, a_{k}\right]$ allows us to write out formulae for $\xi_{k}\left[a_{1}, \ldots, a_{k}\right], \ldots, \xi_{2}\left[a_{1}, \ldots, a_{k}\right]$ in terms of elements from the sets $\Gamma_{k}, \ldots, \Gamma_{2}$, and hence, since $\xi_{1}=1-\xi_{2}-\ldots-\xi_{k}$, to write out a formula for $\xi_{1}\left[a_{1}, \ldots, a_{k}\right]$.

For $k=2$ (see fig. 5) we have

$$
\varepsilon_{2}\left[a_{1}, a_{2}\right]=\left[a_{1}\right] \times\left[a_{2}\right] .
$$

Hence

$$
\xi_{2}\left[a_{1}, a_{2}\right]=\left[a_{1}\right] \times\left[\frac{1}{2} a_{2}\right],
$$

and thereby

$$
\xi_{1}\left[a_{1}, a_{2}\right]=\left(1-\xi_{2}\right)\left[a_{1}, a_{2}\right]=\left[\frac{1}{2} a_{1}, \frac{1}{2} a_{2}\right]-\left[\frac{1}{2} a_{2}, \frac{1}{2} a_{1}\right] .
$$

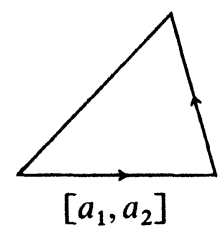

$\left[a_{1}, a_{2}\right]$

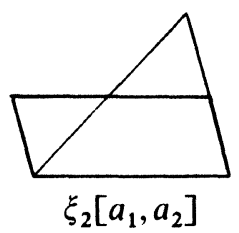

Fig. 5

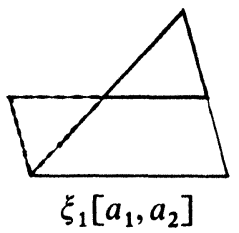

For $k=3$ (see fig. 6) we have

Hence

$$
\begin{aligned}
& \varepsilon_{3}\left[a_{1}, a_{2}, a_{3}\right]=\left[a_{1}\right] \times\left[a_{2}\right] \times\left[a_{3}\right] \\
& \varepsilon_{2}\left[a_{1}, a_{2}, a_{3}\right]=\left[a_{1}\right] \times\left[a_{2}, a_{3}\right]+\left[a_{1}, a_{2}\right] \times\left[a_{3}\right] .
\end{aligned}
$$

$$
\xi_{3}\left[a_{1}, a_{2}, a_{3}\right]=\left[a_{1}\right] \times\left[a_{2}\right] \times\left[\frac{1}{6} a_{3}\right]
$$

and

$$
\begin{aligned}
\xi_{2} & {\left[a_{1}, a_{2}, a_{3}\right]=\left[\frac{1}{2} a_{1}\right] \times \xi_{1}\left[a_{2}, a_{3}\right]+\xi_{1}\left[a_{1}, a_{2}\right] \times\left[\frac{1}{2} a_{3}\right] } \\
& =\left[\frac{1}{2} a_{1}\right] \times\left(\left[\frac{1}{2} a_{2}, \frac{1}{2} a_{3}\right]-\left[\frac{1}{2} a_{3}, \frac{1}{2} a_{2}\right]\right)+\left(\left[\frac{1}{2} a_{1}, \frac{1}{2} a_{2}\right]-\left[\frac{1}{2} a_{2}, \frac{1}{2} a_{1}\right]\right) \times\left[\frac{1}{2} a_{3}\right],
\end{aligned}
$$

and thereby

$$
\begin{aligned}
\xi_{1}\left[a_{1}, a_{2}, a_{3}\right]= & \left(1-\xi_{2}-\xi_{3}\right)\left[a_{1}, a_{2}, a_{3}\right] \\
= & 2\left[\frac{1}{2} a_{1}, \frac{1}{2} a_{2}, \frac{1}{2} a_{3}\right]+\left[\frac{1}{2} a_{1}\right] \times\left[\frac{1}{2} a_{3}, \frac{1}{2} a_{2}\right] \\
& +\left[\frac{1}{2} a_{2}, \frac{1}{2} a_{1}\right] \times\left[\frac{1}{2} a_{3}\right]-\left[a_{1}\right] \times\left[a_{2}\right] \times\left[\frac{1}{6} a_{3}\right] .
\end{aligned}
$$

A simple consideration leads to the simpler expression

$$
\xi_{1}\left[a_{1}, a_{2}, a_{3}\right]=\left[\frac{1}{2} a_{1}, \frac{1}{2} a_{2}, \frac{1}{2} a_{3}\right]+\left[\frac{1}{2} a_{3}, \frac{1}{2} a_{2}, \frac{1}{2} a_{1}\right]-\left[\frac{1}{2} a_{1}\right] \times\left[\frac{1}{2} a_{2}\right] \times\left[\frac{1}{6} a_{3}\right] .
$$

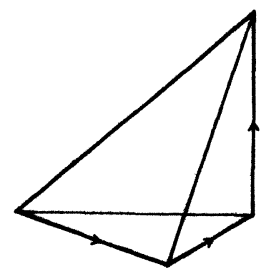

$\left[a_{1}, a_{2}, a_{3}\right]$

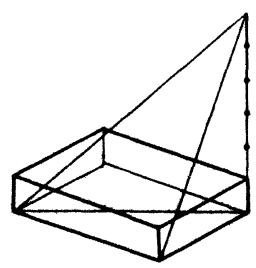

$\xi_{3}\left[a_{1}, a_{2}, a_{3}\right]$

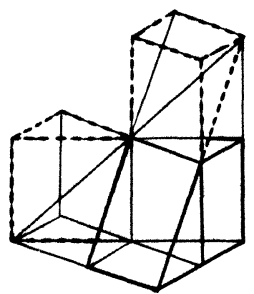

$\xi_{2}\left[a_{1}, a_{2}, a_{3}\right]$

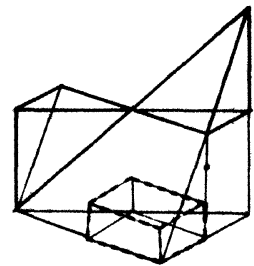

$\xi_{1}\left[a_{1}, a_{2}, a_{3}\right]$

Fig. 6 


\section{The equivalence theorem. Second part.}

We are now in a position to prove that the necessary conditions for translation equivalence in Theorems 1 and 2 are also sufficient. It will be convenient to state this as theorems on the group $\Pi$. Since the necessity of the conditions has been established, we can consider $\mathrm{Vol}_{k}$ and the functions $H_{\phi}$ as functions from $\Pi$ into $L$. With regard to the homomorphism

$$
h\left(A^{k-1}, e^{k-1}\right): \mathfrak{B} \rightarrow \mathfrak{B}\left(A^{k-1}\right) .
$$

it was observed that it defines a homomorphism from $\Pi$ into $\Pi\left(V^{k-1}\right)$, where $V^{k-1}$ is the subspace of $V$ corresponding to $A^{k-1}$, and that this homomorphism is the same for all subspaces $A^{k-1}$ of direction $V^{k-1}$, and all vectors $e^{k-1}$ lying at the same side of $V^{k-1}$, whereas for vectors $e^{k-1}$ lying at different sides of $V^{k-1}$ the homomorphisms differ only by a factor -1 . Denoting by $\overline{V^{k-1}}$ one of the two open halfspaces of $V$ bounded by $V^{k-1}$, we shall denote the homomorphism from $\Pi$ into $\Pi\left(V^{k-1}\right)$ which we obtain by choosing $e^{k-1}$ in $\overline{V^{k-1}}$ by $f\left(\overline{V^{k-1}}\right): \Pi \rightarrow \Pi\left(V^{k-1}\right)$. The sufficiency of the conditions of Theorems 1 and 2 can now for dimension $k$ be expressed as follows:

$1_{k}$. If $x \in \Pi$ satisfies the conditions $\operatorname{Vol}_{k} x=0$, and $f\left(\overline{V^{k-1}}\right) x=0$ for every halfspace $\overline{V^{k-1}}$, then $x=0$.

$2_{k}$. If $x \in \Pi$ satisfies the condition $H_{\Phi}(x)=0$ for all flags $\Phi$, then $x=0$.

As previously noted, the two statements are identical, and true, for $k=1$. It was also noted that $2_{k}$ implies $1_{k}$, since the conditions of $1_{k}$ imply the conditions of $2_{k}$, and that one easily proves by induction that $1_{k}$ implies $2_{k}$. It is therefore sufficient to prove, for $k>1$, that $2_{k-1}$ implies $1_{k}$.

Proof. Let $x \in \Pi$ satisfy the conditions of $1_{k}$, and hence also the conditions of $2_{k}$. Assuming $2_{k-1}$, we must prove that $x=0$.

(a) We write $V=U \oplus V_{*}$, where $U$ is one-dimensional. Let $e \neq 0$ be a vector in $U$, and let $\bar{V}_{*}$ be the open halfspace bounded by $V_{*}$ which contains $e$. Let $A_{*}$ be a $(k-1)$-dimensional subspace of $A$ of direction $V_{*}$, and let $\bar{A}_{*}$ be the open halfspace bounded by $A_{*}$ corresponding to $\bar{V}_{*}$.

Let $\Omega$ be the subgroup of $\Pi$ generated by the elements $d(\lambda)[e] \times y_{*}$, where $\lambda \in L$ and $y_{*} \in \Pi\left(V_{*}\right)$, and let $x: \Pi \rightarrow \Pi / \Omega$ be the canonical map of $\Pi$ onto the quotient group $\Pi / \Omega$ which takes every element of $\Pi$ into the equivalence class of $\Pi$ modulo $\Omega$ to which it belongs.

For every polytope $F$ in some $(k-1)$-dimensional subspace $A^{k-1}$ of $A$ of direction $V^{k-1}$ not containing $e$. and belonging to $\bar{A}_{*}$, we denote by $g(F)$ the polytope in $A$ which is the union of all segments joining a point of $F$ and its 


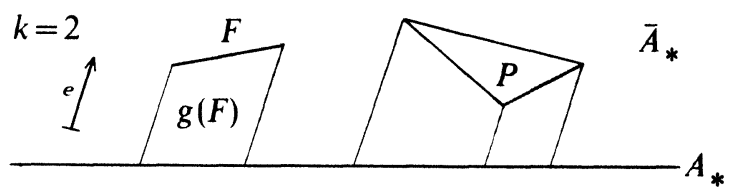

Fig. 7

projection on $A_{*}$ in direction $U$ (see fig. 7). For two such polytopes $F$, which are translates of each other, the corresponding polytopes $g(F)$ are either translates of each other, or one of them is composed of a translate of the other and a prism $I \times R_{*}$, where $I$ is a segment of direction $U$ and $R_{*}$ is a polytope in $A_{*}$. Hence the map $F \mapsto \gamma(F)=\varkappa([g(F)])$ is translation invariant. To a decomposition of $F$ corresponds a decomposition of $g(F)$. Hence the map is also additive. The restriction, that $F$ should lie in $\bar{A}_{*}$, is removed by defining $\gamma(F)$ for an $F$ not in $\bar{A}_{*}$ as the value of $\gamma$ for a translate of $F$ in $\bar{A}_{*}$. Thus the map $F \mapsto \gamma(F)$ defines, for every $V^{k-1}$ not containing $e$, a homomorphism from $\Pi\left(V^{k-1}\right)$ into $\Pi / \Omega$. This homomorphism is also denoted $\gamma$, for all such $V^{k-1}$.

An arbitrary $x \in \Pi$ is represented by an element $X \in \mathfrak{P}$ generated by polytopes in $\bar{A}_{*}$. For every such polytope $P$ we have (see fig. 7)

$$
[P]=\sum \varepsilon[g(F)],
$$

where the sum is extended over all faces $F$ of $P$ whose direction does not contain $e$, and $\varepsilon$ for each $F$ is +1 or -1 according as $F$ is positive or negative with respect to $e$. Hence

$$
x([P])=\sum \varepsilon \varkappa([g(F)])=\sum \varepsilon \gamma(F) .
$$

Using the definition of the functions $f\left(\overline{V^{k-1}}\right): \Pi \rightarrow \Pi\left(V^{k-1}\right)$, we obtain from this the formula

$$
\varkappa(x)=\sum \gamma\left(f\left(\overline{V^{k-1}}\right) x\right),
$$

where the summation is over all halfspaces $\overline{V^{k-1}}$ containing $e$.

Thus, for our $x$, which satisfies the conditions of $1_{k}$, we must have $x(x)=0$, that is to say, we have $x \in \Omega$.

(b) For every generator $d(\lambda)[e] \times y_{*}$ of the group $\Omega$, the element $y_{*} \in \Pi\left(V_{*}\right)$ is generated by products $y_{1} \times \ldots \times y_{i}$, where $y_{1} \in \Xi_{1}\left(V_{* 1}\right), \ldots, y_{i} \in \Xi_{1}\left(V_{* i}\right)$ and $V_{*}=V_{* 1} \oplus \ldots \oplus V_{* i}$. For each such product $y_{1} \times \ldots \times y_{i}$ we can in the product $d(\hat{\lambda})[e] \times y_{1} \times \ldots \times y_{i}$ move the operator $d(\hat{\lambda})$ from the first factor to the second factor. This shows that $d(\hat{\lambda})[e] \times y_{*}$ is of the form $[e] \times z_{*}$, where $z_{*} \in \Pi\left(V_{*}\right)$.

Hence the group $\Omega$ is simply the group of all $[e] \times x_{*}$, where $x_{*} \in \Pi\left(V_{*}\right)$. Thus we have now proved that $x=[e] \times x_{*}$ for some $x_{*} \in \Pi\left(V_{*}\right)$.

(c) In a one-dimensional subspace of $A$ of direction $U$, let $I$ denote a segment 
representing $[e]$. We can use the freedom we have in defining volume (the proportionality factor which is at our disposal) in such a way in $A$ und $A_{*}$ that, when $\mathrm{Vol}_{i *}$ denotes volume in $i$-dimensional subspaces of $A_{*}$, we have

$$
\operatorname{Vol}_{i *}(Q)=\operatorname{Vol}_{i+1}(I \times Q)
$$

for every polytope $Q$ in an $i$-dimensional subspace of $A_{*}$. Then, when $H_{\Phi_{*}}$ : $\Pi\left(V_{*}\right) \rightarrow L$ denotes the Hadwiger function for a flag $\Phi_{*}=\left(V_{*}, \overline{V_{*}^{k-2}}, \ldots, \overline{V_{*}^{i}}\right)$ in $V_{*}$, we have for every $x_{*} \in \Pi\left(V_{*}\right)$

$$
H_{\Phi_{*}}\left(x_{*}\right)=H_{\Phi}\left([e] \times x_{*}\right),
$$

where $\Phi$ is the corresponding flag in $V$ determined by

$$
\Phi=\left(V, \overline{U \oplus V_{*}^{k-2}}, \ldots, \overline{U \oplus V_{*}^{i}}\right),
$$

where $\overline{U \oplus V^{j}}$ means the halfspace of $U \oplus V^{j+1}$ bounded by $U \oplus V^{j}$ which intersects $V_{*}$ in $\overline{V_{*}^{j}}$.

Since our $x=[e] \times x_{*}$ satisfies the conditions of $2_{k}$, we see that $x_{*}$ must satisfy the conditions of $2_{k-1}$. Consequently, $x_{*}=0$, and hence $x=0$.

\section{Additional results.}

It was proved in section 5 (j) that the Hadwiger function $H_{\Phi}: \Pi \rightarrow L$ for an $i$-flag $\Phi$ is a linear function on the vector space $\Pi$, which vanishes on $\Xi_{j}$ for $j \neq i$. It therefore follows from $2_{k}$ that each subspace $\Xi_{i}$ must consist of those $x \in \Pi$ for which $H_{\Phi}(x)=0$ for all $j$-flags $\Phi$ with $j \neq i$. Hence $Z_{i}=\Xi_{i} \oplus \ldots \oplus \Xi_{k}$ must consists of those $x \in \Pi$ for which $H_{\Phi}(x)=0$ for all $j$-flags $\Phi$ with $j<i$. This gives us:

TheOREM 4. An element $X$ of the polytope group $\mathfrak{B}$ belongs to $\mathcal{Z}_{i}$ if and only if $H_{\Phi}(X)=0$ for all $j$-flags $\Phi$ with $j<i$.

For $i=2$, and all $k \geqq 2$, this was proved by $\mathrm{H}$. Hadwiger [6].

Since for every $x \in \Pi$ we have $H_{\phi}(x)=0$ for all but a finite number of flags, we find, using a well-known theorem on vector spaces:

THEOREM 5. All linear functions $H$ from the vector space $\Pi$ into $L$ are determined by the formula $H=\sum c_{\Phi} H_{\Phi}$, where $\Phi \mapsto c_{\Phi}$ is any function from the set of all flags into $L$, and the summation is over all flags.

All linear functions $H_{i}$ from the vector space $\Pi$ into $L$ which vanish on $\Xi_{j}$ for $j \neq i$ are determined by the formula $H_{i}=\sum_{i} c_{\Phi} H_{\phi}$, where $\Phi \mapsto c_{\Phi}$ is any function from the set of all $i$-flags into $L$, and the summation is over all $i$-flags. 
The functions $H=\sum c_{\Phi} H_{\phi}$ are the general Hadwiger functions, and the functions $H_{i}=\sum_{i} c_{\phi} H_{\phi}$ are the general Hadwiger functions of degree $i$. The functions $H_{i}$ are also characterized as those additive functions from $\Pi$ into $L$ which are homogeneous of degree $i$ with respect to the dilation operator.

The only functions $H_{k}$ are the functions $c \mathrm{Vol}_{k}$. Thus the space $Z_{k}=\Xi_{k}$ is onedimensional.

Let $G$ be any group of automorphisms of $A$ which contains the group of translations. Let $\mathfrak{E}_{G}$ denote the subgroup of $\mathfrak{P}$ generated by all elements $P-P_{1}$ $-P_{2}$, where $P$ is composed of $P_{1}$ and $P_{2}$, and all elements $P-Q$, where $Q$ is obtained from $P$ by a transformation in $G$. Two elements $X$ and $Y$ of $\mathfrak{P}$ are called $G$-equivalent and we write $X \stackrel{G}{\sim} Y$, if $X-Y \in \mathfrak{E}_{G}$. The group $\Pi_{G}=\mathfrak{B} / \mathfrak{E}_{G}$ is called the polytope group in $A$ modulo $G$.

One easily shows that the group $I_{G}=\mathfrak{E}_{G} / \mathfrak{E}$ is a linear subspace of $\Pi$. Hence $\Pi_{G}$ is simply the quotient space $\Pi / I_{G}$, and we have

$$
\Pi_{G}=\Xi_{1 G} \oplus \ldots \oplus \Xi_{k G},
$$

where $\Xi_{i G}=\left(\Xi_{i}+I_{G}\right) / I_{G}$. The dilation operator $d(\hat{\lambda})$ may be considered as an endomorphism of $\Pi_{G}$ and has a spectral decomposition similar to the one described in Theorem 3. An element $x$ of $\Pi$ lies in $I_{G}$ if and only if $H(x)=0$ for all general Hadwiger functions $H$ which vanish on $I_{G}$. If we consider the general Hadwiger functions as functions from $\mathfrak{P}$ into $L$, we therefore obtain the following criterion for $G$-equivalence: An element $X$ of the polytope group $\mathfrak{P}$ is $G$-equivalent to 0 , if and only if $H(X)=0$ for all general Hadwiger functions whose restriction to the class of polytopes is $G$-invariant, i.e., for which $H(P)$ $=H(Q)$, when $Q$ is obtained from $P$ by a transformation in $G$.

The groups $G$ of automorphisms of $A$ which contain the group of translations correspond uniquely to the groups of automorphisms of $V$. The following cases are of particular interest.

(i) $G$ is the group corresponding to $\{1, \delta(-1)\}$. In this case $I_{G}$ is the sum of those spaces $\Xi_{i}$ for which $k-i$ is odd. From this we conclude that $X \stackrel{G}{\sim} 0$ for an element $X \in \mathfrak{P}$ if and only if $H_{\phi}(X)=0$ for all $i$-flags for which $k-i$ is even. Cf. H. Hadwiger [3].

(ii) $G$ is the group corresponding to $\left\{\delta\left(\hat{\lambda}^{n}\right) \mid n \in \mathbf{Z}\right\}$, where $\lambda \in L \backslash\{0,1,-1\}$. In this case we have $I_{G}=\Pi$. Hence $X \stackrel{G}{\sim} 0$ for all $X \in \mathfrak{P}$. Cf. V. B. Zylev [21], H. E. Debrunner [2], Ch. Meier [13], H. Hadwiger [9].

Remark. Two polytopes $P$ and $Q$ are called " $G$-zerlegungsgleich", and we write $P \stackrel{G}{\approx} Q$, if there are decompositions $P=P_{1} \cup \ldots \cup P_{n}$ and $Q=Q_{1} \cup \ldots$ $\cup Q_{n}$ such that $Q_{i}$ is obtained from $P_{i}$ by a transformation in $G$ for every $i \in\{1, \ldots, n\}$. Obviously, if $P \underset{\sim}{\approx} Q$, then $P \sim Q$. But conversely, from $P \sim Q$ it follows only that $P$ and $Q$ are "G-ergänzungsgleich", i.e., that there exist 
polytopes $R \cong S$ and $\bar{R} \approx \bar{\sigma} \bar{S}$, such that $\bar{R}$ is composed of $P$ and $R$ and $\bar{S}$ is composed of $Q$ and $S$. However, " $G$-ergänzungsgleich" implies " $G$ zerlegungsgleich", if the ordered field $L$ is archimedean, cf. J.-P. Sydler [19], H. Hadwiger [4], V. B. Zylev [20], V. G. Boltjanskiǐ [1], and Ch. Meier [13].

\section{REFERENCES}

1. V. G. Boltjanskiǐ, Tret'ja problema Gil'berta, Izdatel'stvo "Nauka”, Moskva, 1977.

2. H. E. Debrunner, Zerlegungsähnlichkeit von Polyedern, Elem. Math. 24 (1969), 1-6.

3. H. Hadwiger, Translationsinvariante, additive und schwachstetige Polyederfunktionale, Arch. Math. (Basel) 3 (1952), 387-394.

4. H. Hadwiger, Ergänzungsgleichheit k-dimensionaler Polyeder, Math. Z. 55 (1952), 292-298.

5. H. Hadwiger, Zur Zerlegungstheorie euklidischer Polyeder, Ann. Mat. Pura Appl. (4) 36 (1954), 315-334.

6. H. Hadwiger, Vorlesungen über Inhalt, Oberfläche und Isoperimetrie (Grundlehren Math. Wissensch. 93), Springer-Verlag, Berlin - Göttingen - Heidelberg, 1957.

7. H. Hadwiger, Neuere Ergebnisse innerhalb der Zerlegungstheorie euklidischer Polyeder, Jber. Deutsch. Math.-Verein. 70 (1967/68), 167-176.

8. H. Hadwiger, Translative Zerlegungsgleichheit der Polyeder des gewöhnlichen Raumes, J. Reine Angew. Math. 233 (1968), 200-212.

9. H. Hadwiger, Homothetieinvariante und additive Polyederfunktionen, Arch. Math. (Basel) 25 (1974), 203-205.

10. H. Hadwiger, Zerlegungsgleichheit euklidischer Polyeder bezüglich passender Abbildungsgruppen, Math.-Phys. Semesterber. 22 (1975), 125-133.

11. H. Hadwiger and P. Glur, Zerlegungsgleichheit ebener Polygone, Elem. Math. 6 (1951), 97-106.

12. B. Jessen, Zur Algebra der Polytope, Nachr. Akad. Wiss. Göttingen II 1972, 47-53.

13. Ch. Meier, Zerlegungsähnlichkeit von Polyedern, J. Reine Angew. Math. 253 (1972), 193-202.

14. P. Mürner, Zwei Beispiele zur Zerlegungsgleichheit 4-dimensionaler Polytope, Elem. Math. 29 (1974), 132-135.

15. P. Mürner, Translative Parkettierungspolyeder und Zerlegungsgleichheit, Elem. Math. 30 (1975), 25-27.

16. P. Mürner, Translative Zerlegungsgleichhheit von Polytopen, Arch. Math. (Basel) 29 (1977), 218-224.

17. P. Mürner, G-Zerlegungsgleichheit von Polytopen und homogene G-Parkettierung des ddimensionalen Raumes, Dissertation, Universitäts-Druckerei Bern, 1977.

18. C. H. Sah, Hilbert's third problem, Stony Brook, 1977, not yet in print.

19. J.-P. Sydler, Sur la décomposition des polyèdres, Comment. Math. Helv. 16 (1943-44), 266-273.

20. V. B. Zylev, Equicomposability of equicomplementable figures, Soviet Math. Dokl. 6 (1965), 453-455.

21. V. B. Zylev, G-composedness and G-complementability, Soviet Math. Dokl. 9 (1968), 403-404. 